\title{
Measurement of the Arctic UTLS composition in presence of clouds using millimetre-wave heterodyne spectroscopy
}

\author{
E. Castelli ${ }^{1}$, B. M. Dinelli ${ }^{1}$, S. Del Bianco ${ }^{2}$, D. Gerber ${ }^{3}$, B. P. Moyna ${ }^{3}$, R. Siddans ${ }^{3}$, B. J. Kerridge ${ }^{3}$, and U. Cortesi ${ }^{2}$ \\ ${ }^{1}$ Istituto di Scienze dell'Atmosfera e del Clima, ISAC-CNR, Bologna, Italy \\ ${ }^{2}$ Istituto di Fisica Applicata "Nello Carrara", IFAC-CNR, Sesto Fiorentino, Italy \\ ${ }^{3}$ RAL Space, Harwell Oxford, STFC.RAL, Chilton, UK \\ Correspondence to: E. Castelli (e.castelli@isac.cnr.it)
}

Received: 28 February 2013 - Published in Atmos. Meas. Tech. Discuss.: 28 March 2013

Revised: 25 July 2013 - Accepted: 23 August 2013 - Published: 16 October 2013

\begin{abstract}
MARSCHALS (Millimetre-wave Airborne Receivers for Spectroscopic CHaracterisation in Atmospheric Limb Sounding) is a limb viewing instrument working in the millimetre and sub-millimetre spectral regions (from 294 to $349 \mathrm{GHz}$ ). The scientific rationale of MARSCHALS is the study of the Upper Troposphere and Lower Stratosphere region. In March 2010 MARSCHALS was deployed on-board the M-55 Geophysica stratospheric aircraft during the PREMIER(PRocess Exploration through Measurements of Infrared and millimetre-wave Emitted Radiation)-Ex field campaign. From the campaign base at Kiruna, Sweden, a research flight to investigate the Arctic atmosphere was conducted. For the first time the instrument fully exploited the three spectral bands. In this paper, we present results of the analysis of MARSCHALS measurements acquired during the PREMIER-Ex flight as processed by the Millimetre-wave Atmospheric Retrieval Code. For the second time after the SCOUT-O3 flight in 2005, MARSCHALS measured vertical distributions of temperature, water vapour, ozone and nitric acid over an altitude range of 4 to $24 \mathrm{~km}$. In addition, vertical profiles of carbon monoxide and nitrous oxide were obtained for the first time. The measurements were performed in clear sky conditions and in presence of low and high altitude clouds (that were able to obscure measurements in the middle infrared spectroscopic region) and some information about thick clouds were extracted from the data. The capabilities to derive information on upper tropospheric and lower stratospheric vertical profiles of temperature and minor constituents from millimetre-wave limb sounding observations in the northern polar region are presented and discussed for each of the individual targets. The results of MARSCHALS
\end{abstract}

data analysis contributed to demonstrate the scientific relevance and technical feasibility of millimetre-wave limbsounding of the UTLS proposed for the ESA Earth Explorer 7 candidate Core Mission PREMIER. PREMIER was not selected at the end of the Earth Explorer 7 evaluation process, but it is still being considered for future launch opportunities.

\section{Introduction}

The Upper Troposphere and Lower Stratosphere (UTLS) is a pivotal region of the Earth's atmosphere. In absolute numbers the term UTLS is generally applied to altitude ranges between 4 and $25 \mathrm{~km}$ at polar latitudes and between 8 and $30 \mathrm{~km}$ in the Tropics. Chemical, radiative and dynamical processes that take place at these altitudes have a substantial and mutual influence on surface climate (ESA, 2012 and references therein). In fact, this atmospheric region - due to the very low temperatures of the layers immediately above and below the tropopause height - is where Essential Climate Variables (Mason, 2010) such as surface air temperature and Earth radiation budget are most sensitive to changes in the distribution of radiatively active gases and clouds. Of primary importance in this respect are water vapour and ozone, greenhouse gases which play a key role in the radiative balance of the planet (e.g. Solomon et al., 2010; Hurst et al., 2011; Lacis et al., 1990) and exhibit a large, global-scale, spatio-temporal variability in the UTLS. The distribution of these species, and of other atmospheric constituents in gas-phase and particulates, is ultimately determined by complex interactions between the chemical and 
radiative processes which represent their sources and sinks, as well as the dynamical mechanisms which are responsible for the exchange of air masses between the troposphere and the stratosphere, ranging from planetary or synoptic scales down to the mesoscale (see, for instance, Gettelman et al., 2011 and references therein). In the Arctic region, an accurate representation of the processes that occur in the UTLS is an important prerequisite in understanding the perturbed chemistry in the core of the northern polar vortex, as well as transport and mixing at its boundary. Given that extraordinary events such as the severe ozone depletion observed in the 2011 Arctic winter (January-March 2011, see Arnone et al., 2012 and Manney et al., 2011) are likely to reoccur in the upcoming years, there is a clear need to acquire new observations from space with enhanced vertical and horizontal resolution, which will allow for the investigation of chemical composition, radiative forcing and dynamical transport processes occurring from the outer to innermost region of the vortex. Moreover, the high latitudes of the Northern Hemisphere show clear evidence of the dynamic coupling between the stratosphere and the troposphere, with anomalies in the strength of the polar vortex propagating downward across the lower stratosphere, resulting in circulation anomalies and a perturbation of the Earth's surface climate.

However, to date the UTLS region is not adequately covered by satellite measurements, since the majority of spaceborne sensors are blinded by clouds or - as is the case with the Microwave Limb Sounder (MLS) instrument on-board the Upper Atmosphere Research Satellite (UARS) and Aura platforms (Barath et al., 1993; Waters et al., 2006) or the Sub-Millimetre Receiver (SMR) (Olberg et al., 2003) onboard the Odin satellite (Murtagh et al., 2002) - their instruments are not fully optimised for UTLS measurements in terms of their choice of microwindows (tropospheric penetration), their low vertical resolution and their poor sensitivity (Urban et al., 2005a, b; Sandor et al., 1998; Livesey et al., 2003). To overcome this, as part of the seventh call for Earth Explorer Core missions in the frame of the European Space Agency (ESA)'s Living Planet Programme, one of the three mission candidates that underwent PhaseA feasibility study was the mission "Process Exploration through Measurement of Infrared and Millimetre-wave Emitted Radiation" (PREMIER). PREMIER was not selected at the end of the evaluation process. Nonetheless, the strong scientific case and the technical feasibility demonstrated by Phase-A preparatory studies laid the basis to seek for a new launch opportunity either through national initiatives or in response to ESA Earth Explorer 9 Call for Ideas. The primary scientific objective of the PREMIER mission was to gain a better understanding of the interaction processes linking atmospheric chemistry and dynamics with climate. This imply the investigation of distribution and transport of trace gases and of the radiative effects of water vapour and clouds in the mid- to upper troposphere and lower stratosphere. This can be achieved through the combined observations by two limb-sounders: one of which operating in the thermal infrared (InfraRed Limb Sounder, IRLS), the other in the sub-millimetre-wave region (Millimetre-Wave Limb Sounder (MWLS) a.k.a. Stratosphere-Troposphere Exchange and Climate Monitor Radiometer, STEAM-R). To demonstrate the merits of the mission concept, an airborne demonstrator for the MWLS instrument on PREMIER a.k.a. MARSCHALS (Oldfield et al., 2001) - was built. Respective infra-red instruments to demonstrate the IRLS capabilities are already existing, e.g. Michelson Interferometer for Passive Atmospheric Sounding - STRatospheric aircraft (MIPAS-STR, Piesch et al., 1996), Gimballed Limb Observer for Radiance Imaging of the Atmosphere, AirBorne version (GLORIA-AB, Friedl-Vallon et al., 2006). MARSCHALS is designed to sample the atmosphere using a limb-viewing geometry from a stratospheric balloon or aircraft. It has already been deployed from the M-55 Geophysica high altitude aircraft during the SCOUT-O3 campaign in 2005 (Dinelli et al., 2009).

As part of the preparatory studies, demonstration and testing activities carried out in parallel to PREMIER Phase-A, a series of field campaigns (PREMIER-Ex) for the deployment of airborne precursors of PREMIER instrumentation on-board the high altitude research platform M-55 Geophysica was performed in 2009-2010. The PREMIER-Ex activities consisted of a test campaign, which took place at midlatitude (Oberpfaffenhofen, Germany, Lat. $48.1^{\circ} \mathrm{N}$, Lon. $11.3^{\circ} \mathrm{E}$ ) in November 2009 and in a scientific campaign, performed in the Arctic region (Kiruna, Sweden, Lat. $67.8^{\circ} \mathrm{N}$, Lon. $20.4^{\circ} \mathrm{E}$ ) in March 2010 in the frame of the RECONCILE (Reconciliation of essential process parameters for an enhanced predictability of Arctic stratospheric ozone loss and its climate interactions) project. The MARSCHALS instrument was present on-board the M-55 for both flights. While for the first science campaign (SCOUT-O3, 2005) only one of the three spectral bands of MARSCHALS was operational, all three bands have now been implemented for PREMIER-Ex. However, because of unfavourable flight conditions, which were inherent to the test campaign in 2009, only the data from the scientific campaign in Kiruna are worth investigating.

In this paper, we present the results of MARSCHALS measurements obtained during the PREMIER-Ex scientific campaign as processed by MARC (Millimetre-wave Atmospheric Retrieval Code). Since all the three spectral bands were operating properly, we could retrieve $\mathrm{CO}$ and $\mathrm{N}_{2} \mathrm{O}$ vertical profiles in addition to the temperature, $\mathrm{HNO}_{3}, \mathrm{O}_{3}$ and $\mathrm{H}_{2} \mathrm{O}$ profiles that had already been measured using the single operating band in the SCOUT-O3 campaign. The structure of the paper is as follows: in Sect. 2 we describe the MARSCHALS instrument and its new features; in Sect. 3 we present the PREMIER-Ex campaign and the flight of 10 March 2010; a description of MARSCHALS data analysis is presented in Sect. 4; results, discussion 
and intercomparisons with other instruments are reported in Sect. 5 and in Sect. 6 conclusions are given.

\section{The MARSCHALS spectrometer}

\subsection{Instrument description}

MARSCHALS uses a vertically scanning antenna of $235 \mathrm{~mm}$ diameter to measure thermal emission from the atmosphere. The limb of the atmosphere is sampled through an open aperture in the starboard side of the Geophysica aircraft at angles equivalent to $1 \mathrm{~km}$ tangent point steps. A single scan of 28 measurements starts just below ground level and reaches up to just above platform altitude and includes an additional space-view at $20^{\circ}$ above horizontal. An active antenna control loop with a gyro as its reference stabilises the antenna to within fractions of a degree stability $\left( \pm 0.01^{\circ}\right.$ when the absolute roll angle rate of change is contained within the $\pm 0.63^{\circ} \mathrm{s}^{-1}$ design range). Because of various biases in the antenna knowledge (i.e. non-reproducibility of the alignment w.r.t. the airframe, temporal bias in the gyro record due as a function of geographic latitude, etc.) the antenna system has to be regularly reset to horizontal. If during these resets the aircraft is not flying level - which it quite often does not then these updates can lead to the following scan can be offset in angle range compared to the nominal scan angles. This is mostly compensated by the safety margin in the nominal scan angle range, and it is not a problem for the data analysis because the absolute value of the tangent point altitude can be accurately reconstructed post-flight, but it implies that in reality tangent points of neighbouring scans will be at slightly different altitudes.

The duration of a single scan is $\sim 3 \mathrm{~min}$. Integration time at each view angle is 4 times $250 \mathrm{~ms}$. Incoming radiation is coupled to each of three millimetre-wave heterodyne radiometers centred at 300,325 and $345 \mathrm{GHz}$, where it is down-converted to the range $12-24 \mathrm{GHz}$, amplified, and detected using a single channeliser spectrometer. Observation time is shared between the three spectral bands by means of alternating atmospheric scans. The total spectral bandwidth of the channeliser spectrometer is $12 \mathrm{GHz}$, split up in 60 channels of $200 \mathrm{MHz}$ channel bandwidth (the Intermediate Frequency (IF) bandwidth of the receivers is less than $12 \mathrm{GHz}$ to a varying degree). The signal due to aliased radiances is filtered out by a high performance single sideband filter (one for each radiometer) which reduces these signals by at least $30 \mathrm{~dB}$.

The antenna scan system employs a dedicated Inertial Measurement Unit (IMU) to improve the control of the pointing direction. The antenna control loop measures the instrument (aircraft) roll angle with high precision and corrects for it in the loop. This makes the instrument line-of-sight extremely stable. To help in assessing the impact of cirrus clouds on the observations, MARSCHALS incorporates an
Optical Cloud Monitor (OCM). The spectroscopic characterisation of the three MARSCHALS bands are summarised in Table 1 (the bands' frequency range reported in the table follow the original instrument configuration). Additional information about the MARSCHALS instrument can be found in Dinelli et al. (2009), where the configuration used during the SCOUT-O3 campaign is presented together with a rendering of the instrument and its subsystems.

\subsection{UAMS instrument updates}

The first scientific deployment of MARSCHALS during the SCOUT-O3 campaign took place before the instrument was operating according to its final specifications (only the $300 \mathrm{GHz}$ band was operating nominally during the remote sensing flight). In order to address this, a comprehensive instrument upgrade and characterisation project "Upgrades to Airborne Millimetre-wave Sounder" (UAMS) took place between SCOUT-O3 and PREMIER-Ex. Following is a list of points addressed by the UAMS project.

\subsubsection{Single Sideband Filter (SSBF)}

New Single Sideband Filters were developed for the three MARSCHALS bands by the Queen's University of Belfast. All of them have both good image rejection $(>30 \mathrm{~dB})$ and low signal insertion loss $(<0.5 \mathrm{~dB})$. The new filters for band $\mathrm{B}$ and $\mathrm{D}$ are a free-standing 3-layer linear slot-array type Frequency Selective Surface (FSS). The band C filter is a freestanding 3-layer dual polarisation annular slot array type FSS.

\subsubsection{Band B receiver}

The original band B receiver, supplied for MARSCHALS from a separate ESA technology development programme, had unsatisfactory noise performance $(>20000 \mathrm{~K}$ in single sideband (SSB)) and a pronounced gain slope in the IF passband. The entire receiver was replaced by a design developed by RAL and EADS Astrium (UK). The new receiver employs a simplified single-stage down-conversion scheme which eliminates the IF gain slope. In addition, a new mixer has been specifically designed for this channel. It has been designed to have inherently better performance in the signal sideband and includes an integral IF preamplifier, thereby improving IF match and IF noise temperature. The feedhorn used on the new receiver is a scaled version of the design developed for band C (and band D) by the University of Navarra. A new doubler has also been developed for this channel. The noise temperature of the new receiver, measured with a broadband power metre, is listed in Table 1 . The gain slope has also been measured and was shown to have decreased from $\sim 10 \mathrm{~dB}$ to less than $1 \mathrm{~dB}$. Figure 1 shows the position of the band $\mathrm{B}$ receiver in the new layout after the UAMS update. 
Table 1. Frequency coverage and spectral performance of the three MARSCHALS spectral bands. The signal band dimensions reported in the table refer to the original instrument configuration.

\begin{tabular}{llll}
\hline & Band B & Band C & Band D \\
\hline 1st LO frequency [GHz] & 317.46 & 337.48 & 360.80 \\
IF band [GHz] & $12.0-23.6$ & $12.0-21.0$ & $12.0-18.6$ \\
Signal band [GHz] & $293.86-305.46$ & $316.48-325.48$ & $342.20-348.80$ \\
Image band [GHz] & $329.46-341.06$ & $349.48-358.48$ & $372.80-379.40$ \\
Spectral bandwidth [GHz] & 11.66 & 9.20 & 6.80 \\
Channel bandwidth [GHz] & 0.2 & 0.2 & 0.2 \\
Channels used [No.] & 44 & 40 & 32 \\
Main target species & $\mathrm{O}_{3}$ & $\mathrm{H}_{2} \mathrm{O}(325.2 \mathrm{GHz})$, & $\mathrm{CO}(345.79 \mathrm{GHz})$, \\
& & $\mathrm{O}_{3}$ & $\mathrm{HNO}_{3}, \mathrm{O}_{3}$ \\
$T$ sys before upgrade [K] & 19000 & 18000 & 19000 \\
$T$ sys after upgrade [K] & 6000 & 10000 & 9000 \\
Sideband filter & linear slot FSS & dual polarisation & linear slot FSS \\
Sideband suppression & $>30 \mathrm{~dB}$ & annular slot FSS & \\
Signal insertion loss & $<0.5 \mathrm{~dB}$ & $<30 \mathrm{~dB}$ & $>30 \mathrm{~dB}$ \\
\hline
\end{tabular}

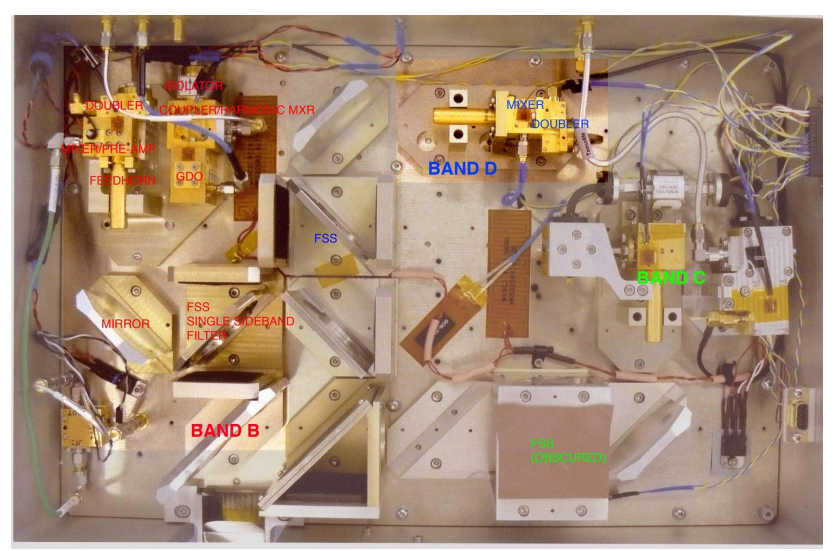

Fig. 1. Constellation of the MARSCHALS receivers and sideband filters after the UAMS instrument upgrades. The atmospheric signal enters the quasioptical subsystem at the bottom of the picture. The band $\mathrm{D}$ signal is separated by a polariser, the band $\mathrm{B}$ and $\mathrm{C}$ signals are separated by a beam splitter.

\subsubsection{Band C receiver}

The components in the band $\mathrm{C}$ receiver were unchanged (only the FSS single sideband filter was replaced). However, it was slightly repackaged to accommodate changes in the physical layout of the band D receiver. After the FSS upgrades the receiver performance was improved, but the improvements were less than expected. After several iterations of components, the subharmonic mixer and doubler were both replaced, producing further improvements in the noise temperature. The final value is listed in Table 1 . The position of band $\mathrm{C}$ receiver is shown in Fig. 1.

\subsubsection{Band D receiver}

Prior to the deployment of MARSCHALS in SCOUT-O3 in Darwin, the band D receiver exhibited a SSB noise temperature of approximately $19000 \mathrm{~K}$. During the deployment in Darwin the channel failed and no data was obtained; this was ascribed to a failed doubler in the local oscillator chain. The mixer originally installed in this band had a noise temperature of $\sim 2000 \mathrm{~K}$ Double Side-Band (DSB). This has now been replaced with a new mixer with a noise temperature of approximately $1500 \mathrm{~K}$ DSB. The failed doubler has been substituted with a new RAL doubler incorporating a RAL Schottky diode. The noise temperature of the new receiver is listed in Table 1. The layout of the instrument after the UAMS project and the position of the receiver are shown in Fig. 1 .

\subsubsection{Instrument Control Unit (ICU)}

The ICU stack has been fitted with external console connectors to allow field diagnostics without stack disassembly (as was necessary in the SCOUT-O3 campaign). A number of software modifications has also been performed. An error in the reading of the aircraft roll-angle data has been corrected. The aircraft roll-angle data is used to periodically calibrate the inertial navigation system of MARSCHALS. In the SCOUT-O3 campaign incorrectly read aircraft data could offset the antenna pointing; this is now not the case anymore. Roll-angle updates were hardcoded to beginning of a full 3band measurement cycle. However, to gain spectral performance the duration of a full set of atmospheric measurements has now increased from $\sim 1 \mathrm{~min}$ to $\sim 10 \mathrm{~min}$. To make up for that, the option for a "commandable roll-angle update" has 
been implemented in the ICU software, which permits more regular updates to the aircraft roll-angle to mitigate pointing angle drifts.

\subsubsection{Antenna pointing system}

The antenna pointing motor and electronics are exposed systems in the open instrument bay of the aircraft, where temperature and humidity variations between the stratosphere and ground conditions can be dramatic. There were some reliability issues with the inductosyn ${ }^{\mathrm{TM}}$ interface board during the SCOUT-O3 campaign, so this system has also been replaced by a completely rebuilt Antenna Pointing System module. This has so far operated with $100 \%$ reliability both in laboratory tests and in flight conditions.

\subsubsection{Thermal stability}

Outside temperatures at $20 \mathrm{~km}$ flight altitude can be as low as $-90^{\circ} \mathrm{C}$. This puts a lot of strain on the temperature stability control system. This is addressed by increasing the wattage of the existing heaters on the radio frequency components, as well as adding a 4th channel to the thermal stabilisation control unit to allow the calibration hot load to be stabilised. In addition to that passive thermal insulation has been added to various subsystems. The cryostat bay and the calibration hot load have been enclosed to reduce convective cooling. The receiver modules, local oscillator modules, phase lock modules and the channeliser have all been fitted with insulating styrofoam padding.

\subsubsection{Instrument characterisation}

The significant changes to the instrument performed under UAMS required a number of instrument characterisation measurements to be repeated. Most importantly these included measurements of the antenna function (field of view), the spectral response functions (instrument line shape) and the spectral performance of the receivers (standing waves, linearity, frequency calibration).

The Azimuthally Collapsed Antenna Patterns (ACAP) and polarisation have been measured in an anechoic chamber at Queen Mary University in London. Analogous measurements have been performed in the past, but these repeated tests are of higher quality because they are performed at higher angular resolution and because of the improved spectral performance of the instrument. The measurement setup has also been better shielded from standing waves in the path between the test signal source and the detector. The ACAP and the polarisation have been measured for all three spectral bands individually and are used in the data analysis process. We have also determined the marginal line-of-sight offsets between the three bands and have corrected for them in the calculation of the scan angles.

The spectral response functions have been measured in the laboratory at RAL. As with the antenna characterisation, a much improved setup compared to the previous measurements has been applied. A line is injected into the $12-24 \mathrm{GHz}$ IF port using a coupler (and amplification to compensate for the coupler loss) so that the spectral response of the instrument is measured against an input comprising a swept continuous wave signal from the synthesiser superimposed on a broadband noise source (the receiver front end viewing an ambient blackbody load).

The use of isolators at the input and output of the line injection system means that it can be removed from the system without significantly affecting the match between the receivers and IF (and hence the spectral response of the system under test). A first set of spectral response measurements had been performed before the PREMIER-Ex campaign.

Initial test results showed that most spectral channels were stable, however as was observed in previous spectral response tests a small proportion of channels were unstable. These channels showed noticeable fluctuations in spectral response between measurement runs. After initial tests, the location of the unstable channels relative to the spectral lines of interest was investigated. The conclusion was that a reordering of the channeliser units was advantageous, enabling the unstable channels to be moved to spectral areas of lower importance. The re-ordering of the channeliser units was completed and additional spectral response tests were undertaken. More details about the channel re-ordering can be found in Spang et al. (2012).

The $200 \mathrm{MHz}$ spectral resolution of the MARSHALS channeliser does not allow the detection of high-frequency standing waves in the system. To check for the presence of standing waves, the University of Bremen kindly lent RAL an Observatoire de Paris-Meudon (ARPEGES-LED) wideband Acousto-Optic Spectrometer (AOS). The unit was the result of the ESA funded Wideband AOS WP2411 and has a nominal bandwidth of $1.5 \mathrm{GHz}$ and channel spacing of $0.89 \mathrm{MHz}$. Within the limitations of the noise performance of the receivers, no standing waves have been detected. More detailed results from the instrument characterisation, as well as accounts on other minor system tests can be found in Moyna et al. (2010).

\section{PREMIER-Ex scientific campaign}

\subsection{Campaign overview}

The aim of the PREMIER mission was to quantify the processes that control the composition and structure of the UTLS. The PREMIER-Ex activities culminated in a scientific campaign in the northern polar region in March 2010. The scientific objectives addressed by the PREMIER-Ex campaign were the quantification of horizontal mixing processes in the UTLS and of the cirrus formation processes in the Arctic atmosphere. The PREMIER-Ex scientific flight (hereafter denoted as PrEx 1) was performed on 10 March 
2010. During the first week of March the vortex was located above the Siberian Arctic and then moved backward towards the Scandinavian Peninsula. On 10 March the polar vortex was located above Scandinavia. The PrEx 1 flight track was planned so as to sound the vortex filamentation on the vortex boundary, and thus to probe the horizontal mixing of vortex and mid-latitude air. An additional constraint was to find a match with A-Train satellites (e.g. CALIPSO, Aura) and ENVISAT overpasses for validation purposes. Using European Centre for Medium-range Weather Forecasts (ECMWF) assimilated forecast model data and results from the Chemical Lagrangian Model of the Stratosphere (CLaMS), the flight path was planned going to the West, as shown in the flight path reported in Fig. 2a and then going back to Kiruna with a triangular flight pattern. The PrEx 1 flight started at $\sim 07: 20$ UTC and finished at $\sim$ 10:30 UTC, while the A-Train satellite overpasses were performed at about 10:20 (MODIS)/10:40 (MLS) and 12:20 (MLS) UTC and the ENVISAT overpass was performed at about 08:45 UTC. Precise temporal coincidence with correlative satellite data is therefore only available for the first two legs of the flight. The second PREMIER-Ex flight, which was supposed to study processes related to Arctic cirrus formation and planned for the 13 March 2010, could not be performed due to a severe aircraft engine problem.

In Fig. 2a we report the values of the Potential Vorticity extracted from the ECMWF archive in Potential Vorticity Unit (PVU, $1 \mathrm{PVU}=10^{-6} \mathrm{Km}^{2} \mathrm{~s}^{-1} \mathrm{~kg}^{-1}$ ) at $125 \mathrm{hPa}$ with respect to latitude and longitude in the flight region. In the same plot we report the M-55 flight track (in black) together with the position of the MARSCHALS tangent points for each scan (in grey). The red line marks the region with $\mathrm{PV}$ equal to $14 \mathrm{PVU}$, that can be considered as the outer border of the vortex at this pressure level (Mariotti et al., 1997; Christensen et al., 2005). Comparing the position of MARSCHALS tangent points with PV values we can notice that the flight was divided into three legs: the first two legs enabled MARSCHALS Lines-of-Sight (LOS) to look partially inside the vortex, while the third to look at air masses from mid-latitude and vortex filamentation.

\subsection{MARSCHALS measurements during PREMIER-Ex scientific flight of 10 March 2010}

As seen in Sect. 2.2, the UAMS upgrades produced an improvement of the noise performances. Additionally the system reliability was improved, which meant that the scan sequence could be speed up by removing redundant integration cycles at each view. This has allowed to increase the number of limb-scans per flight hour and to obtain a better horizontal sampling rate with respect to the SCOUT-O3 flight. During the PrEx 1 flight, MARSCHALS collected 56 limb scans in the time interval from 07:24 UTC to 10:27 UTC. Each band was acquired in a separate scan, and the three bands were acquired in CBD sequence. In total 19 scans were
Table 2. Frequency range and retrieval targets of the three MARSCHALS bands. The frequency ranges of the MARSCHALS bands reported in the table, used for the PrEx 1 data analysis, follow the updated instrument characterisation.

\begin{tabular}{lll}
\hline Band & Frequency range & Target retrieval quantities \\
\hline B & $296.760-305.360$ & $\begin{array}{l}\mathrm{O}_{3}, \mathrm{~N}_{2} \mathrm{O}, \mathrm{HNO}_{3}, \\
\text { external continuum }\end{array}$ \\
C & $317.780-325.380$ & $\begin{array}{l}\text { temperature, } \mathrm{H}_{2} \mathrm{O}, \mathrm{O}_{3}, \\
\mathrm{HNO}_{3}, \text { external continuum }\end{array}$ \\
D & $341.900-348.100$ & $\begin{array}{l}\mathrm{O}_{3}, \mathrm{HNO}_{3}, \mathrm{CO}, \\
\text { external continuum }\end{array}$ \\
\hline
\end{tabular}

recorded in band B, 19 in band C and 18 in band D. During the flight MARSCHALS collected Level 1 data of good quality for all the three bands. This is therefore the first time that MARSCHALS band B and D measurements could be analysed to produce Level 2 data. Table 2 reports the frequency range where the three MARSCHALS bands have good performances and that were therefore used for the analysis reported in this paper.

Figure $2 b$ shows the altitude of the tangent points of each scan given as a function of the acquisition time. The figure shows that there are two parts of the flight (from scans 6 to 31 and from scans 35 to 53) where the aircraft altitude was nearly constant. Figure $2 \mathrm{~b}$ also shows that there was an uneven altitude coverage of the three bands during the flight. This affects the quality of the retrieval products, because of the different vertical distribution of the information content of each scan. The few scans that had a reduced altitude coverage or were acquired during changes of direction of the aircraft were not used in the analysis.

\section{MARSCHALS data analysis}

In this section, we briefly describe the MARC retrieval code, the new features introduced in the retrieval code with respect to the version previously used for the SCOUT-O3 data analysis and we describe the results of the analysis of MARSCHALS spectra.

\subsection{Retrieval code}

The PREMIER-Ex MARSCHALS data analysis was performed using the MARC code (Carli et al., 2007). The MARC retrieval code was developed under an ESA contract for the analysis of the calibrated and geolocated spectra measured by MARSCHALS. It makes use of the global-fit (Carlotti, 1988), multi target (Dinelli et al., 2004) techniques and uses Optimal Estimation (OE) or maximum a posteriori approach (Rodgers, 2000) and the Levenberg-Marquardt algorithm (Levenberg, 1944; Marquardt, 1963) during the iterative retrieval procedure to reduce the step width and to speed up the convergence to the minimum of the cost function. 

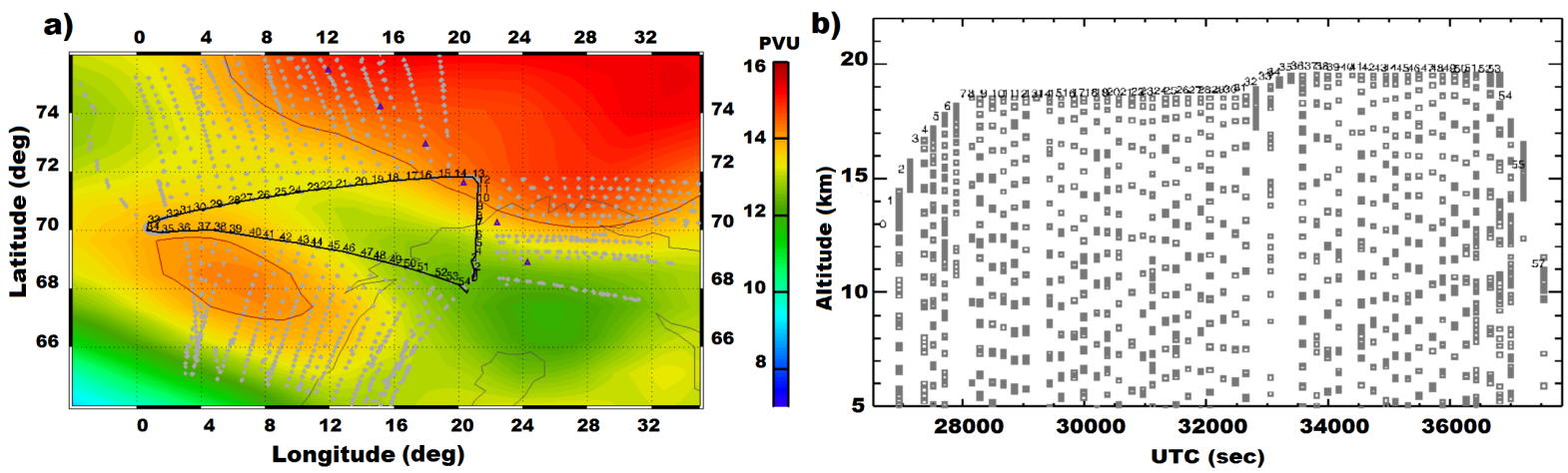

Fig. 2. (a) PV in PVU obtained from the ECMWF archive at $125 \mathrm{hPa}$ as a function of latitude and longitude in the flight region. Black line marks the flight track during the PrEx 1 flight, the position of MARSCHALS tangent points of each scan is shown in grey, while blue triangles represents the position of MLS/Aura scans. (b) Position of MARSCHALS measurements for band B, band C and band D with respect to altitude and time for the PrEx 1 flight.

A detailed description of the main features of the code is provided in Carli et al. (2007) and a description of its use for the analysis of real data can be found in Dinelli et al. (2009). A description of the parametrization of the atmosphere and of the atmospheric line shape used into the MARC code can be found in Bianchini et al. (2008).

MARC retrieves vertically distributed quantities (such as temperature and Volume Mixing Ratios (VMRs) of gases whose emission lines are present in MARSCHALS bands) and scalar quantities, in the form of one value for each analysed scan. The Forward Model (FM) internal to the retrieval code uses a state of the art molecular continuum model developed together with a specific spectroscopic database (Kuhn et al., 2003) and uses an additional (retrievable) continuum profile to account for the continuum like contribution of clouds opaque in the observed frequency region. Originally the retrievable scalar quantities were: the pointing bias (a correction term that enables to account for eventual instrumental mispointings) and the gain and offset (used to correct possible calibration errors).

For the PREMIER-Ex data analysis the possibility to retrieve a frequency shift has been implemented into the MARC code. The frequency shift is retrieved as a frequency offset applied to the Instrument Line Shape (ILS), and is a single scalar number for each scan, because it is assumed to be due to instrumental characterisations only. The frequency shift assumes positive values when the simulated spectrum has to be shifted toward higher frequencies. We tested the retrieval of the frequency shift on simulated data obtaining satisfactory results (Cortesi et al., 2012).

Along with the profiles of the target species and the instrumental parameters, the MARC algorithm provides also retrieval diagnostics that can be divided into scan diagnostic and target diagnostic. The scan diagnostic quantities are: the reduced $\chi^{2}$, the information content (both defined as in Rodgers, 2000) and the trace of the full Averaging Kernel (AK) matrix (which gives information on the total degrees of freedom of the retrieval). The target diagnostic quantities are: the biased error, that is the combination of the retrieval error due to the noise with the a priori error, the AKs of each target, and the individual information content quantifier. These quantifiers have already been discussed in Dinelli et al. (2009), Carli et al. (2007). For this analysis we have also computed the vertical resolution of the retrieval products in the form of the Full Width at Half Maximum (FWHM) of the AK using the expression given by Ridolfi and Sgheri (2009).

\subsection{MARSCHALS retrieval strategy}

The retrieval strategy used for the inverse processing of MARSCHALS measurements during the PrEx 1 flight was optimised through a dedicated approach. As a starting point we applied the strategy used for the data analysis performed on the SCOUT-O3 data and described in Dinelli et al. (2009). However, the different geographic location of the measurements, the upgrades of the instrument and the presence of all three bands for the first time implied that most of the adopted choices had to be revised.

Because the noise characterisation of the new instrument setup was more reliable, in the current analysis we used the noise level as reported in the Level 1B files, that provide a measured value for every single spectral channel. In addition the new FOV and ILS functions, measured with the strategy described in Sect. 2.2 were used.

Each MARSCHALS scan was analysed individually, retrieving four scalar instrumental parameters (gain, offset, pointing bias angle and frequency shift) along with vertical distributions of temperature, water, ozone, nitric acid, nitrous oxide, carbon monoxide and extinction coefficient. Because of the different spectral coverage of the three bands, the vertically resolved targets are scan dependent. Table 2 reports the list of the vertically resolved targets of each band. The profiles above and below the highest and lowest retrieved grid points have been extrapolated following the shape of 
the initial profiles (scaled with the highest and the lowest retrieved point, respectively), while negative VMR values are not allowed into the MARC code.

As already mentioned in Sect. 4.1, the MARC code uses the OE method. Therefore, it is important to correctly define both the a priori information of the atmospheric status and its uncertainty. Temperature, pressure, $\mathrm{O}_{3}$ and $\mathrm{H}_{2} \mathrm{O}$ a priori data (used also as initial guess profiles) were obtained from ECMWF Meteorological Archival and Retrieval System (MARS) archive, using the operational data on 91 model levels. The a priori data for all the other gases like $\mathrm{N}_{2} \mathrm{O}$, $\mathrm{CO}$ and $\mathrm{HNO}_{3}$ were taken from the IG2 database (Remedios et al., 2007) that contains climatological averaged profiles and 1-sigma variabilities for the four seasons and for 5 latitudinal bands. The IG2 1-sigma variability was used as a priori uncertainty for all the targets. The lower limit of this uncertainty was set to a target dependent threshold, expressed as percentage of the a priori profile. This threshold was $100 \%$ for $\mathrm{O}_{3}$ and $\mathrm{H}_{2} \mathrm{O}$ while it was set to $50 \%$ for $\mathrm{N}_{2} \mathrm{O}, \mathrm{HNO}_{3}$ and $\mathrm{CO}$. The a priori error used for temperature was a constant value of $3 \mathrm{~K}$ that is the estimated error of ECMWF data. The a priori error on the external continuum was set to a constant value of $0.2 \times 10^{27} \mathrm{~cm}^{2}$. The profiles of the other gases, not retrieved during the analysis, are extracted from the IG2 polar winter atmosphere. During the revision of this paper we realised that the IG2 data for $\mathrm{CH}_{3} \mathrm{Cl}$ were overestimated. $\mathrm{CH}_{3} \mathrm{Cl}$ emission in band $\mathrm{D}$ is very close to that of $\mathrm{CO}$ and a wrong profile may badly affect its retrieval. Therefore, the $\mathrm{CH}_{3} \mathrm{Cl}$ VMR profile below $20 \mathrm{~km}$ was inferred from MLS/Aura data measured in polar regions on the same date of the flight.

A series of preliminary tests were performed in order to find the best retrieval strategy that could be used for the fit. These tests showed that temperature and $\mathrm{H}_{2} \mathrm{O}$ can be retrieved from band $\mathrm{C}$ measurements only. However, because the initial guess profiles of $\mathrm{H}_{2} \mathrm{O}$ are very different from the real atmospheric conditions and the profiles retrieved from band $\mathrm{C}$ are sometimes unstable, $\mathrm{H}_{2} \mathrm{O}$ is still included into the band $\mathrm{B}$ and $\mathrm{D}$ retrievals to account for the contribution given by the far wings of its lines to the analysed spectrum. The profiles obtained in the analysis of these bands are not included into the retrieval products. Therefore, the devised retrieval strategy was to start the analysis from a band $\mathrm{C}$ scan $(\operatorname{scan} N)$. For the two subsequent scans, $N+1$ in band $\mathrm{B}$ and $N+2$ in band $\mathrm{D}$, we used the temperature value retrieved from scan $N$ and we retrieved $\mathrm{H}_{2} \mathrm{O}$ (with the other targets) using as a priori the profile retrieved from scan $N$. The minimum altitude of the used observations, as well as the optimal target dependent retrieval grids, were chosen after some dedicated tests, using the quantifiers described in Sect. 4.1 to find the best retrieval set up. All observations with tangent altitudes above the ground level were retained in the analysis. The used retrieval altitude grids, common to all the scans of PrEx 1 flight are listed (in km) below:
- temperature: $24,20,18,17,15,13,11,9,7,5$

- $\mathrm{H}_{2} \mathrm{O}: 24,21,18,17,16,15,14,13,12,11,10,9,8,7$, 6,4

- $\mathrm{O}_{3}: 24,21,18,17,16,15,14,13,12,11,8,5$

- $\mathrm{HNO}_{3}: 23,20,17,16,14,12,9,6$

- $\mathrm{N}_{2} \mathrm{O}: 20,16,14,12,10,8$

- CO: $21,17,13,7$

- external continuum: 18, 17, 16, 15, 14, 13, 12, 11, 10, $9,8,7,6$

As for the SCOUT-O3 analysis, one or two retrieval grid points have been added above the highest flight altitude in order to reduce the errors due to the assumed vertical distribution of the targets above the highest retrieval point.

\subsection{MARSCHALS retrieval diagnostic}

Table 3 shows the averaged Degrees Of Freedom (DOF) obtained in the analysis of the three MARSCHALS bands for all vertically resolved targets. We see that the three MARSCHALS bands contain an evenly distributed information on $\mathrm{O}_{3}$ and on the external continuum, while for $\mathrm{HNO}_{3}$ the best information is contained in band $\mathrm{C}$ and $\mathrm{D}$. The values reported in Table 3 confirm that the highest information on $\mathrm{H}_{2} \mathrm{O}$ is in band $\mathrm{C}$, and show that for $\mathrm{N}_{2} \mathrm{O}$ and $\mathrm{CO}$ the number of retrieved DOF is close to the number of altitudes of their retrieval grid. A comparison of Table 3 with Fig. 7 of Dinelli et al. (2009) shows that the number of DOF obtained for band $\mathrm{C}$ in this flight for $\mathrm{H}_{2} \mathrm{O}, \mathrm{O}_{3}$ and $\mathrm{HNO}_{3}$ have increased. This is due to both the improvements of the MARSCHALS instrument and the lower tropopause altitude in the geographical region where the flight took place. In fact the SCOUT-O3 campaign was performed in Darwin, at tropical latitudes, and the tropopause altitude was always higher than the flight altitude, while in this flight the tropopause was always below the aircraft.

The values of the reduced $\chi^{2}$ obtained in the analysis are reported in Fig. 3a, where the blue, black and red symbols are used for the scans of band B, C and D, respectively. We see that the $\chi^{2}$ value is on average around 1.4, apart from few scans in the third leg of the flight. Band B always presents slightly higher $\chi^{2}$ values than the other two bands. As an example of the quality of the retrieval and of the instrument performances, the bottom panels of Fig. 3 report the values of the AKs trace and of the information content for each scan using the same colour code. As expected the three bands have different information content due to both the different number of targets and the covered spectral region. Band $\mathrm{C}$ always has higher DOF and information content. The lower information content and AK trace value found for some scans is due to their reduced altitude coverage (see Fig. 2b). 


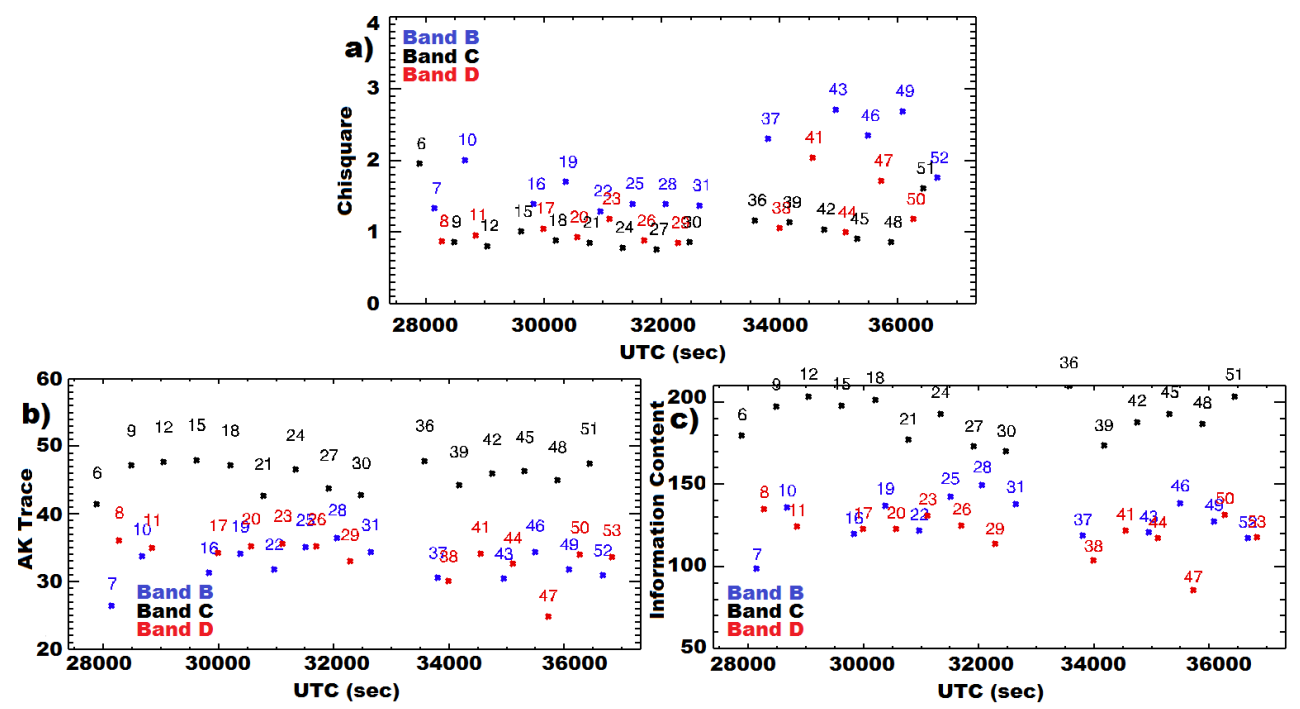

Fig. 3. (a) Retrieved reduced $\chi^{2}$ for PrEx 1 flight for each scan as a function of time. (b) Trace of AK matrix for each scan as a function of time. The length of the state vectors is 59 elements in band B, 63 in band C and 57 in band D. (c) Information content for each scan as a function of time. Blue for band B, black for band $\mathrm{C}$ and red for band $\mathrm{D}$.

Table 3. Degrees of Freedom of the three MARSCHALS bands for the vertically resolved targets.

\begin{tabular}{lccccccc}
\hline Band & Temperature & $\mathrm{H}_{2} \mathrm{O}$ & $\mathrm{O}_{3}$ & $\mathrm{HNO}_{3}$ & $\mathrm{~N}_{2} \mathrm{O}$ & $\mathrm{CO}$ & Cont. \\
\hline B & - & 2.8 & 10.8 & 3.2 & 5.2 & - & 6.5 \\
C & 4.5 & 15.0 & 10.2 & 6.4 & - & - & 5.6 \\
D & - & 3.6 & 10.0 & 6.7 & - & 3.2 & 5.9 \\
\hline
\end{tabular}

In order to better evaluate the retrieval performances, in Fig. 4 we show the average residuum together with corresponding standard deviation and average noise level for two scans in each MARSCHALS band: For one scan we obtained good $\chi^{2}$ values (scan 22 in band B, scan 12 in band $\mathrm{C}$ and scan 29 in band $\mathrm{D}$, residuum plotted in blue) while for the other we had worse performances (scan 49 in band $\mathrm{B}$, scan 51 in band $\mathrm{C}$ and scan 47 in band $\mathrm{D}$, plotted in red). As can be noticed, worst $\chi^{2}$ values were obtained when the residuum standard deviation was higher than the noise level. In general no particular feature can be seen in the residuum. The residuum behaviour highlights the quality of the MARSCHALS measurements and retrieval results.

\section{Results and discussion}

In this section we present and discuss the results obtained for the instrumental parameters and the vertically resolved quantities from the analysis of MARSCHALS data during the PREMIER-Ex campaign on 10 March 2010.

The retrieved values of the instrumental parameters offset, gain, pointing and frequency shift stay constant along the flight indicating that the instrument did not show problems
Table 4. Average scalar quantities retrieved from the three MARSCHALS bands.

\begin{tabular}{lrrrr}
\hline Band & $\begin{array}{r}\text { Offset } \\
{[\mathrm{K}]}\end{array}$ & Gain & $\begin{array}{r}\text { Pointing } \\
\text { bias [deg] }\end{array}$ & $\begin{array}{r}\text { Frequency } \\
\text { shift [MHz] }\end{array}$ \\
\hline B & $0.42 \pm 0.78$ & $0.98 \pm 0.01$ & $-0.037 \pm 0.071$ & $10.1 \pm 0.6$ \\
C & $-0.71 \pm 0.70$ & $1.00 \pm 0.01$ & $-0.013 \pm 0.032$ & $13.0 \pm 1.2$ \\
D & $-1.47 \pm 0.68$ & $0.99 \pm 0.01$ & $-0.003 \pm 0.024$ & $11.1 \pm 2.3$ \\
\hline
\end{tabular}

of degradation along the flight. The retrieved values of the scalar quantities, averaged over all the results of the different bands, are shown in Table 4. Offset values are very close to zero, and gain values are close to 1 , indicating good radiometric performances of the instrument. The small values assumed by the pointing bias indicates that the a-posteriori pointing knowledge is very good. Finally the values obtained for the frequency shift are of the order of few $\mathrm{MHz}$, largely below the instrumental frequency step $(200 \mathrm{MHz})$.

All the results obtained for vertically resolved quantities will be presented using colour maps where the examined quantity is mapped as a function of altitude and of acquisition time. We report on separate panels the retrieved quantities, the retrieval error, the individual information content and the vertical resolution (FWHM). We recall here that the individual information content is not an absolute quantifier, but it represents a relative quantity that shows how much the a priori error has been reduced during the analysis. A value of the information content of 0.5 represents a reduction of the a priori error of $\sqrt{2}$ (Dinelli et al., 2009). Retrieved values with individual information content very low are strongly biased towards the a priori. 

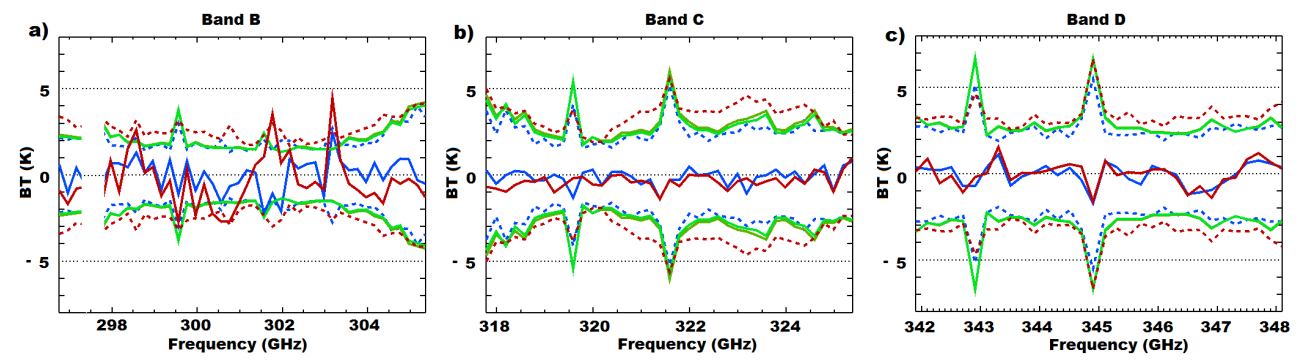

Fig. 4. Average noise level (green), average residuum (solid lines, blue for the best and red for the worse scan) and corresponding standard deviations (dashed lines) for: (a) scans 22 blue and 49 red in band B, (b) scans 12 in blue and 51 in red in band C, (c) scans 29 in blue and 47 in red for band D.
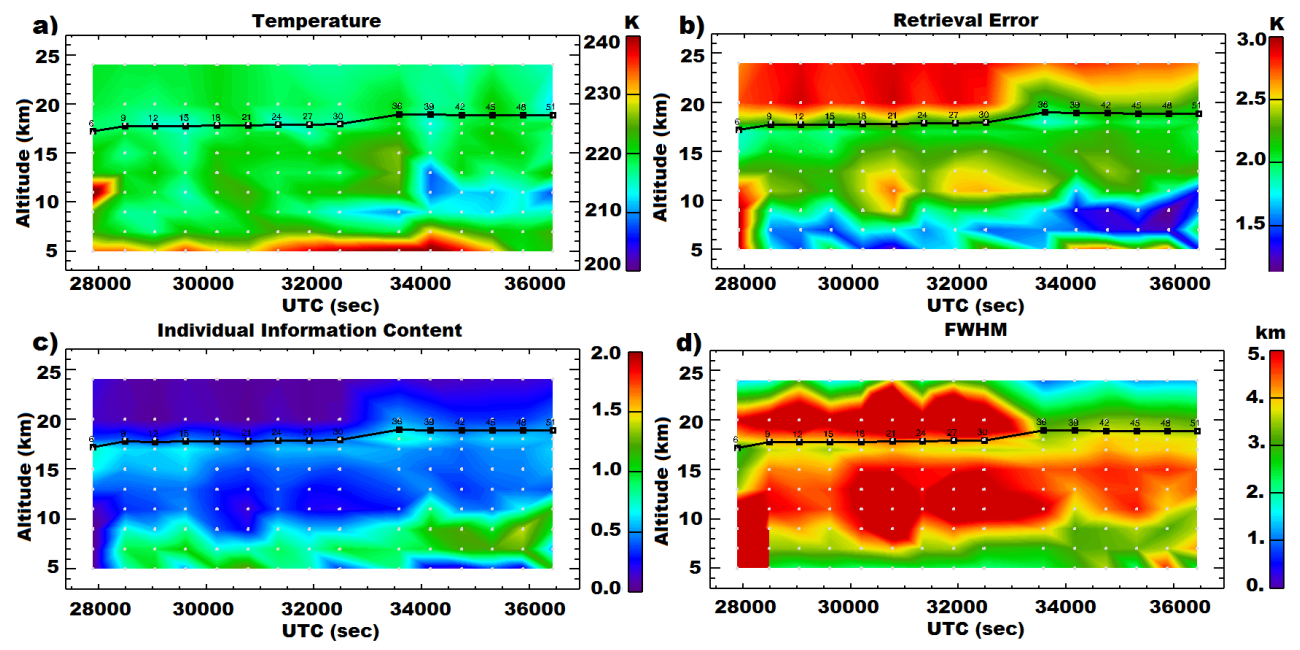

Fig. 5. Maps of retrieved values and quantifiers plotted as a function of altitude and acquisition time. The black line is the aircraft altitude and the black dots mark every single scan. White dots mark the used retrieval grid points. (a) Temperature retrieved from band $\mathrm{C}$ scans, (b) its retrieval (biased) error, (c) the individual information content and (d) the vertical resolution (FWHM).

The results are discussed in one subsection for each retrieval target. The obtained results will also be validated through the comparison with the vertical profiles obtained by MLS/Aura or MIPAS/ENVISAT satellite sensors.

In the validation exercise, the coincidence criteria applied was $4 \mathrm{~h}$ maximum time delay and $200 \mathrm{~km}$ maximum geographical distance. The use of MLS/Aura profiles retrieved with a tomographic (2-D) approach (Livesey et al., 2006) (taking into accounts horizontal inhomogeneities) is particularly appropriate for the comparison with MARSCHALS data during the PrEx 1 flight, where horizontal mixing and vortex filamentation were under investigation. For the same reason we prefer to use the MIPAS2D database (Dinelli et al., 2010), instead of the ESA Level 2 MIPAS dataset, because it has been obtained with a full 2-D analysis code as well.

\subsection{Temperature}

Temperature profiles retrieved from band $\mathrm{C}$ are presented in Fig. 5. As already said in Sect. 4.2, temperature has been retrieved from band $\mathrm{C}$ scans only. However, the information content on temperature is very low, confirming the findings of the previous analysis (Dinelli et al., 2009). During the flight the temperature profiles are homogeneous in the altitude range from 24 to $12 \mathrm{~km}$, while below $12 \mathrm{~km}$, where the value of the individual information content becomes higher, temperature shows a minimum in the third leg of the flight.

The quality of the retrieved temperature data can be evaluated comparing MARSCHALS results with MIPAS/ENVISAT coincident data. Results of the comparison between MARSCHALS and MIPAS/ENVISAT temperature profiles show a general good agreement. In particular, low temperature values at about $12 \mathrm{~km}$ are present also in MLS/Aura data in correspondence of the third leg of the flight (see Cortesi et al., 2012).

\subsection{Water vapour}

The results obtained for $\mathrm{H}_{2} \mathrm{O}$ in the analysis of band $\mathrm{C}$ scans are presented in Fig. 6 together with the information content and vertical resolution. As can be noticed the information content distribution is not homogeneous because of 

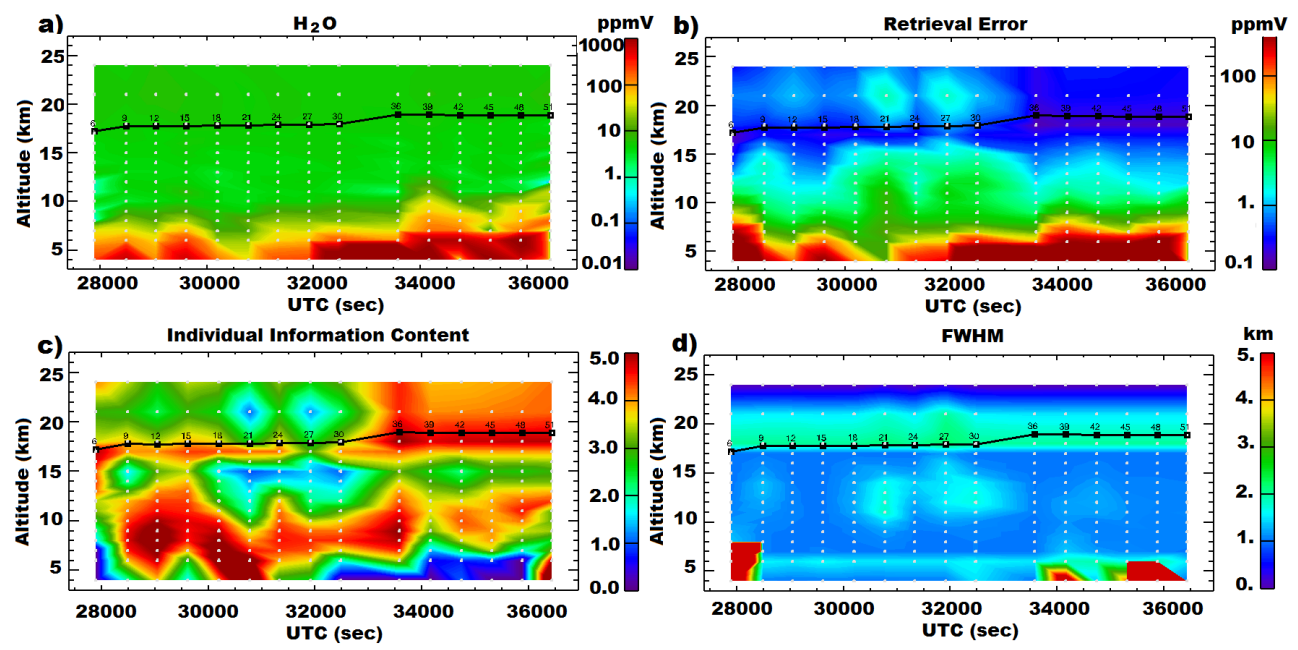

Fig. 6. As in Fig. 5 but for $\mathrm{H}_{2} \mathrm{O}$ retrieved from band $\mathrm{C}$ scans only.
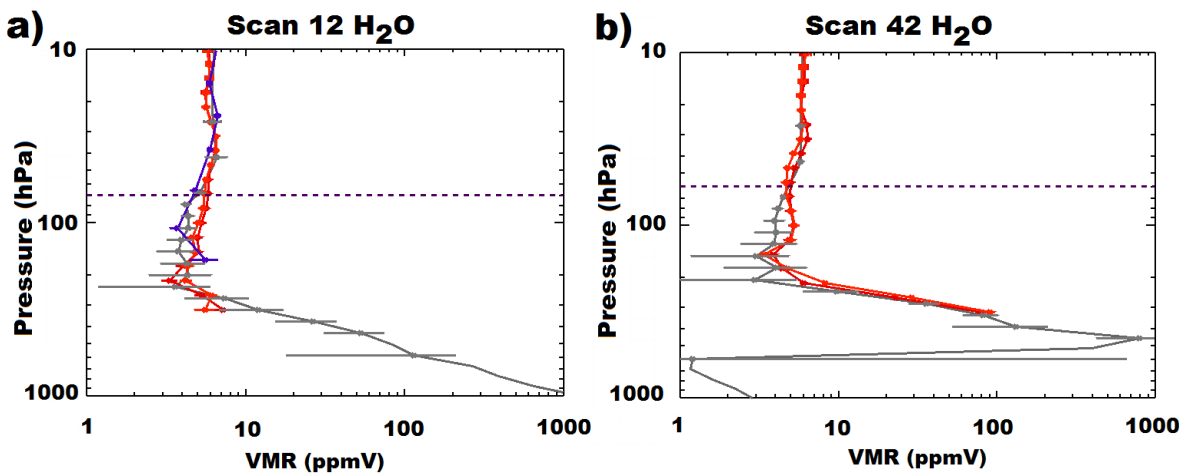

Fig. 7. Comparison of $\mathrm{H}_{2} \mathrm{O}$ profiles retrieved from MARSCHALS scans 12 and 42 (grey) with MIPAS/ENVISAT (blue) and MLS/Aura (red) $\mathrm{H}_{2} \mathrm{O}$ profiles. The horizontal dashed line represents the flight altitude level.

the uneven altitude coverage of the MARSCHALS measurements (see Fig. 2b). In particular, we have low information below $8 \mathrm{~km}$ in the second half of the flight and in the altitude range from 14 to $16 \mathrm{~km}$ from scan 21 to 30 . The retrieved VMR does not show particular features, apart from an enhancement at low altitudes in the second half of the flight that justifies the lower information content found (higher $\mathrm{H}_{2} \mathrm{O}$ VMRs imply more opaque spectra).

Comparison between water vapour retrieved profiles for MARSCHALS scans with MLS/Aura and MIPAS/ENVISAT coincident profiles show a very good agreement with MLS data especially around the hygropause as can be noticed in Fig. 7, where we report an example for MARSCHALS scans 12 and 42.

\subsection{Ozone}

$\mathrm{O}_{3}$ can be retrieved from the measurements of all the three bands (band B, C and D). The obtained results for single scan analysis are reported in Fig. 8. The individual information content of the three bands is always high, although the best performances are obtained for band B measurements and the worst for band $\mathrm{D}$. The lower values are always found in the altitude region between 15 and $10 \mathrm{~km}$. In any case, the individual information content of $\mathrm{O}_{3}$ during $\mathrm{PrEx} 1$ is high over a wider altitude range with respect to what was found during the SCOUT-O3 data analysis. This is certainly due to the different geolocation of the flight, but also due to the improvements of the instrument.

In Fig. 9, we compare $\mathrm{O}_{3}$ retrieved profiles for scans from 10 to 12 with MLS/Aura and MIPAS/ENVISAT coincident profiles and we find a generally good agreement. In particular, $\mathrm{O}_{3}$ profiles retrieved from band $\mathrm{B}$ and $\mathrm{D}$ (scan 10 and 11 , respectively) show good agreement with satellite data. No particular features can be seen in MARSCHALS $\mathrm{O}_{3}$ profiles during the flight. 

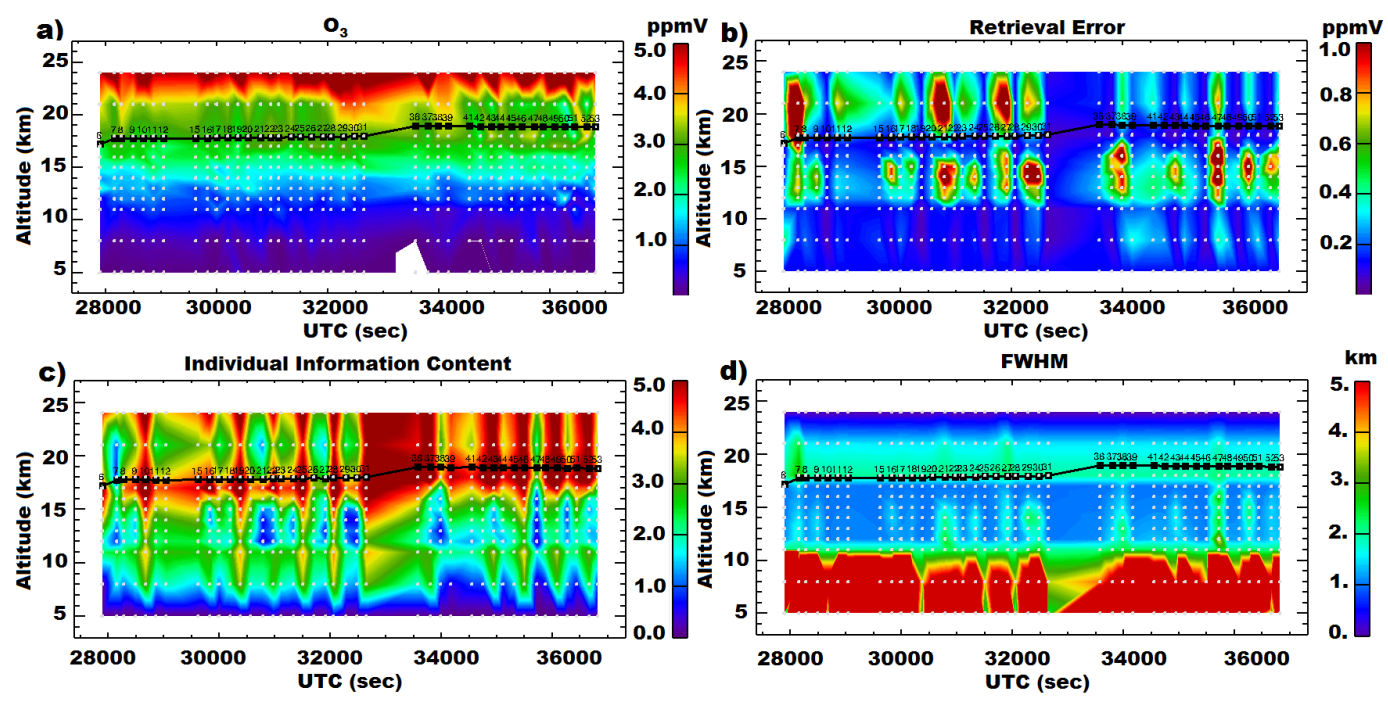

Fig. 8. As in Fig. 5 but for $\mathrm{O}_{3}$ retrieved from all bands.

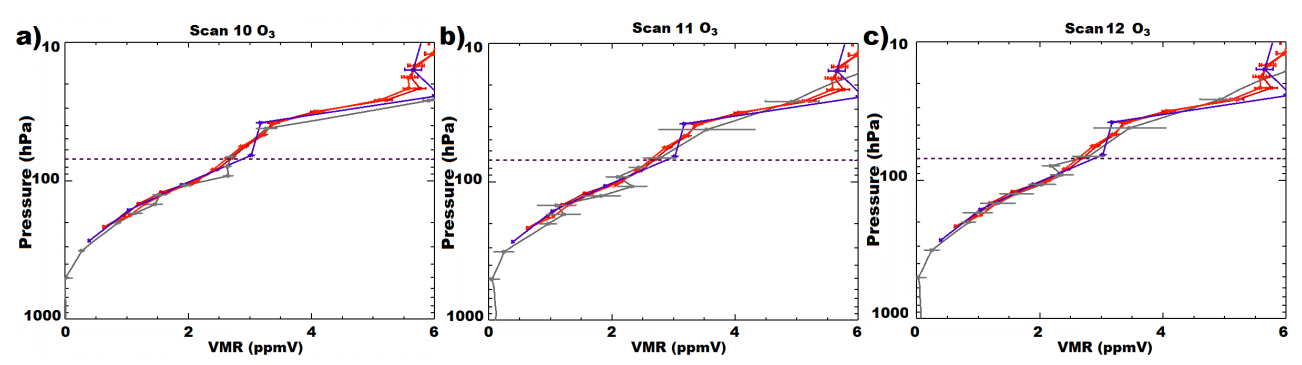

Fig. 9. Comparison of $\mathrm{O}_{3}$ profiles retrieved from MARSCHALS scan 10 to 12 (grey) with MIPAS/ENVISAT (blue) and MLS/Aura (red) $\mathrm{O}_{3}$ profiles. The horizontal dashed line represents the flight altitude level.
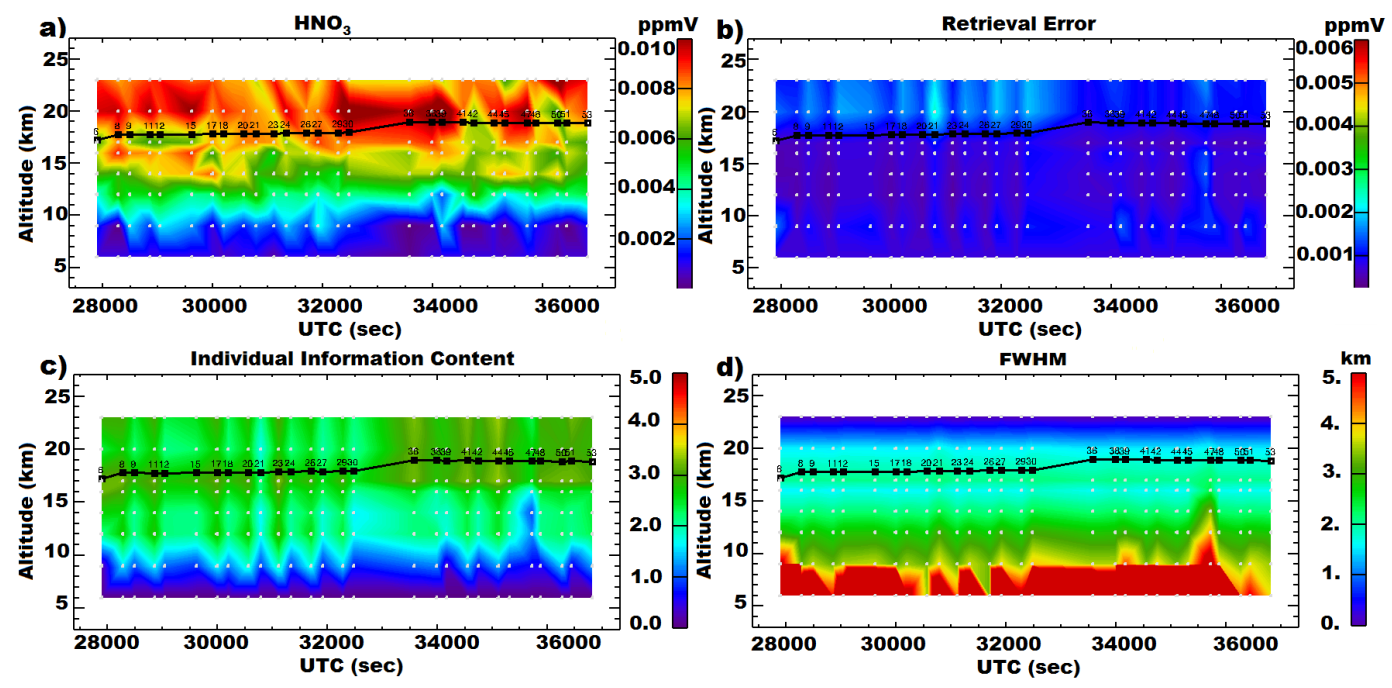

Fig. 10. As in Fig. 5 but for $\mathrm{HNO}_{3}$ retrieved from band $\mathrm{C}$ and D. 

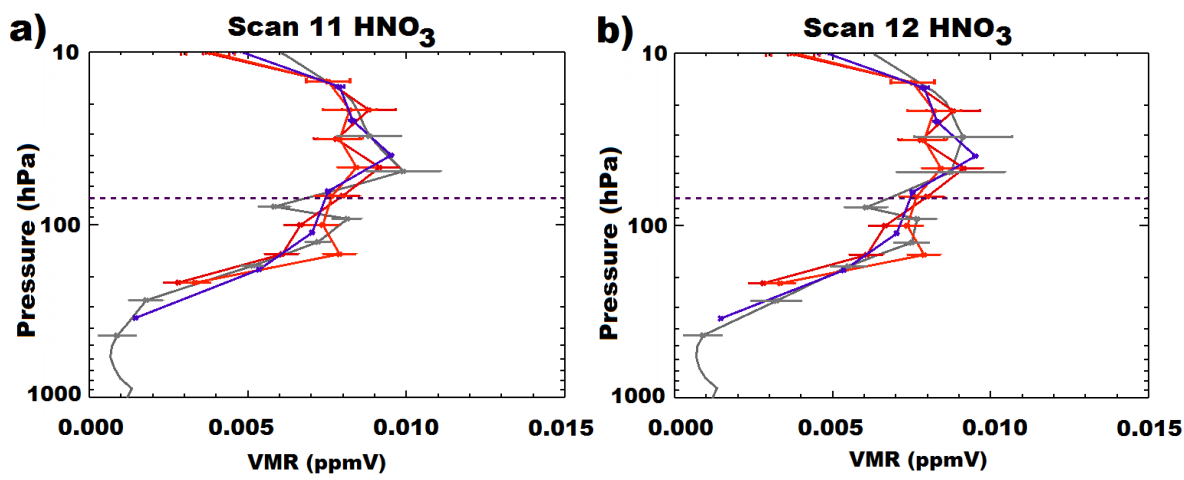

Fig. 11. Comparison of $\mathrm{HNO}_{3}$ profiles retrieved from MARSCHALS scans 11 and 12 (grey) with MIPAS/ENVISAT (blue) and MLS/Aura (red) $\mathrm{HNO}_{3}$ profiles. The horizontal dashed line represents the flight altitude level.
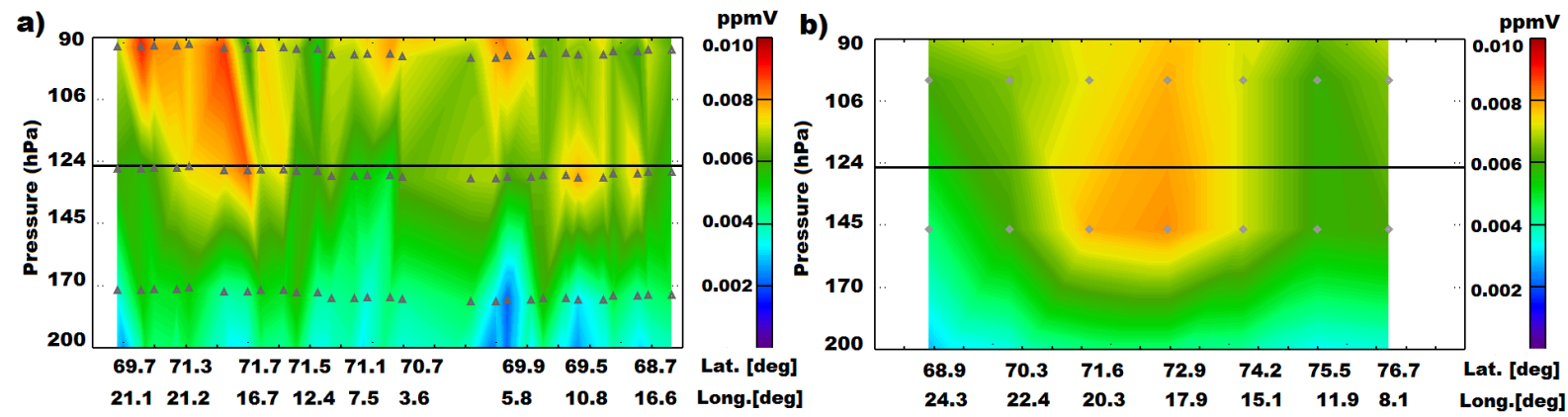

Fig. 12. (a) $\mathrm{HNO}_{3}$ values retrieved from MARSCHALS as a function of pressure (from 90 to $200 \mathrm{hPa}$ ) and latitude and longitude of the instrument during the flight. The black line corresponds to the $125 \mathrm{hPa}$ level while grey points represent the MARSCHALS retrieval grid points. (b) $\mathrm{HNO}_{3}$ MLS/Aura values as a function of pressure and latitude, longitude for the scans marked with triangles in Fig. $2 \mathrm{a}$.

\subsection{Nitric acid}

In Fig. 10, we present the results obtained for the retrieval of $\mathrm{HNO}_{3}$ profiles from band $\mathrm{C}$ and band $\mathrm{D}$ data. The profiles obtained from band $\mathrm{B}$ data are not reported since the information on $\mathrm{HNO}_{3}$ contained in this band was not sufficient to produce significant results. $\mathrm{HNO}_{3}$ values retrieved by MARSCHALS show the presence of a filament of polar air with higher $\mathrm{HNO}_{3}$ values, between 14 and $16 \mathrm{~km}$.

An example of the comparison of $\mathrm{HNO}_{3}$ data retrieved from MARSCHALS for scan 11 in band D and scan 12 in band $\mathrm{C}$ and coincident $\mathrm{HNO}_{3}$ profiles from MLS/Aura, and MIPAS/ENVISAT is reported in Fig. 11. In general we can notice a very good agreement.

Looking at Fig. 2a, we can notice that MARSCHALS limb scans from 11 to 20 approximately sounded the Arctic polar vortex, while scans from 36 to 47 explored a filament of vortex air, and finally scans from 6 to 10 from 21 to 35 and from 48 to 54 were performed outside the vortex. This behaviour is reflected in $\mathrm{HNO}_{3}$ values retrieved by MARSCHALS at about $125 \mathrm{hPa}$, reported in Fig. 12a with respect to pressure and latitude, longitude of the instrument during the flight (where the black line marks the
$125 \mathrm{hPa}$ level) with higher $\mathrm{HNO}_{3}$ values found in correspondence of vortex air and filament of vortex air. The coincident MLS/Aura $\mathrm{HNO}_{3}$ values along the orbit track between $68^{\circ} \mathrm{N}$ and $76^{\circ} \mathrm{N}$ latitude crossing the first two flight legs (Fig. 12b) show a similar behaviour at $125 \mathrm{hPa}$ (marked by the black line), thus confirming MARSCHALS results. The capability of the MARSCHALS instrument to resolve the filament of polar vortex air is crucial for the investigation of horizontal mixing processes in the UTLS atmospheric region.

\subsection{Nitrous oxide}

$\mathrm{N}_{2} \mathrm{O}$ profiles can be retrieved only from band $\mathrm{B}$ data, where few $\mathrm{N}_{2} \mathrm{O}$ spectral lines are present near one of the ozone lines. The obtained results are reported in Fig. 13. As can be seen in the figure, the information content of MARSCHALS band $\mathrm{B}$ is not particularly high, but it is sufficient to enable the MARC code to retrieve the $\mathrm{N}_{2} \mathrm{O}$ VMR values. Due to the choice we made to use relatively large a priori errors, in order to impose a weak constrain on the measurements, the obtained $\mathrm{N}_{2} \mathrm{O}$ profiles are sometimes oscillating.

The comparison of $\mathrm{N}_{2} \mathrm{O}$ profiles retrieved from MARSCHALS and MIPAS/ENVISAT profiles and MLS/Aura show a quite good agreement, especially 

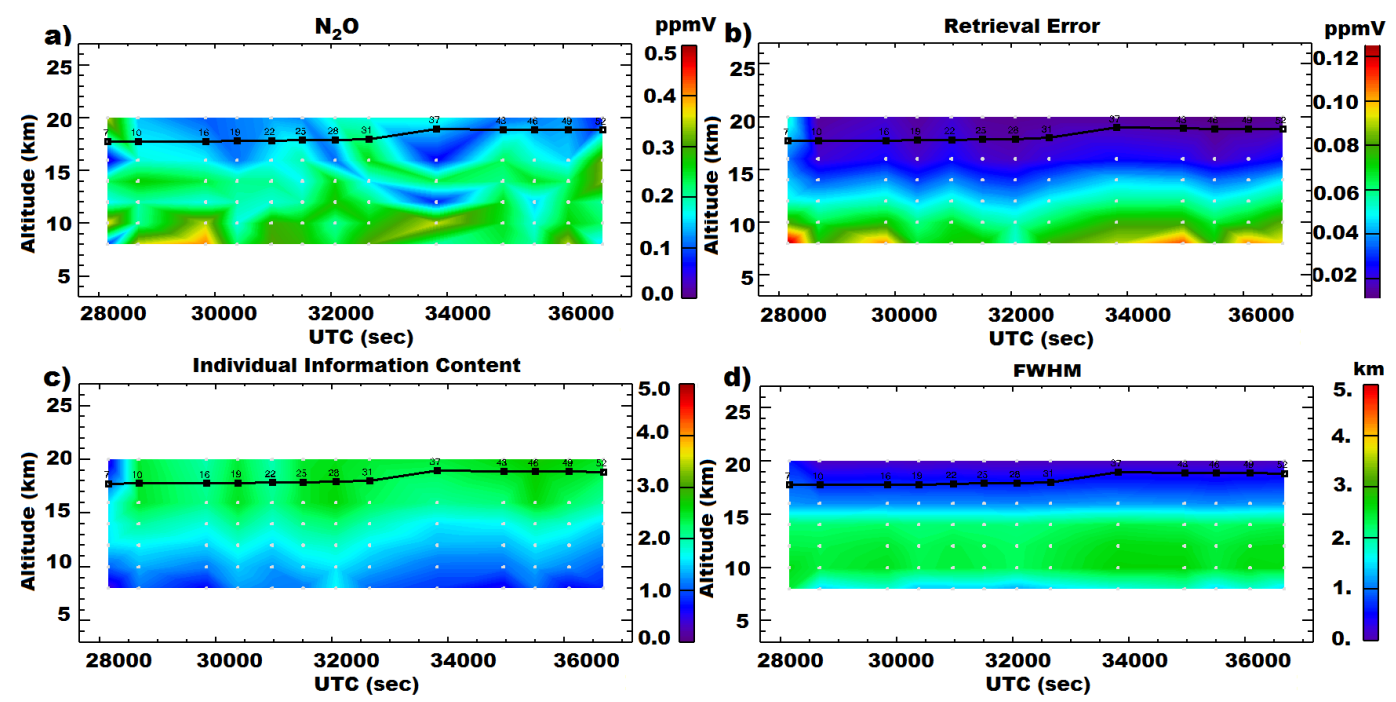

Fig. 13. As in Fig. 5 but for $\mathrm{N}_{2} \mathrm{O}$ retrieved from band $\mathrm{B}$.

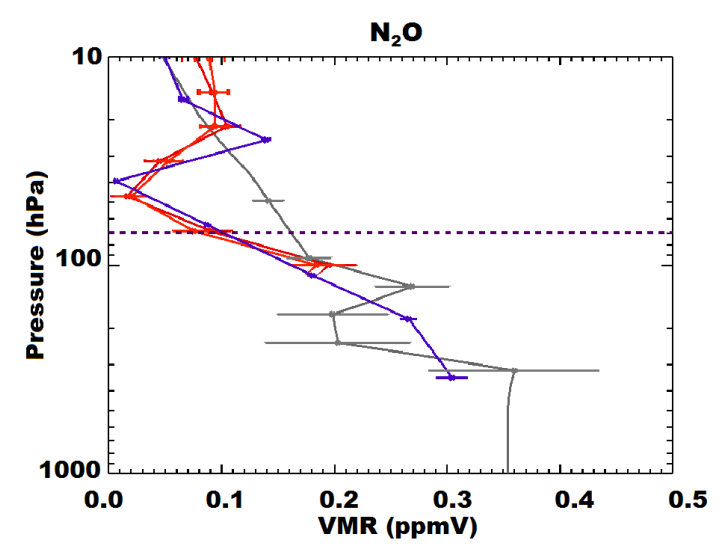

Fig. 14. Comparison of $\mathrm{N}_{2} \mathrm{O}$ profile retrieved from MARSCHALS scan 10 (grey) with MIPAS/ENVISAT (blue) and MLS/Aura (red) $\mathrm{N}_{2} \mathrm{O}$ profiles. The horizontal dashed line represents the flight altitude level.

at low altitude (see Fig. 14 for scan 10) even if the MARSCHALS $\mathrm{N}_{2} \mathrm{O}$ profiles show some oscillations with respect to other instruments. Since few $\mathrm{N}_{2} \mathrm{O}$ spectral lines are present into MARSCHALS band $\mathrm{B}$, the obtained results can be considered a proof of the robust analysis performed on MARSCHALS data of the PREMIER-Ex flight from Kiruna.

\subsection{Carbon monoxide}

CO has only one spectral feature in band D and therefore it can be retrieved from the measurements of that band. Very close to its emission there are few $\mathrm{CH}_{3} \mathrm{Cl}$ spectral lines. These lines badly interfere with the $\mathrm{CO}$ emission at low altitudes, where the pressure broadening is quite large. As already said in Sect. 4.2 , the $\mathrm{IG}_{2}$ profile of $\mathrm{CH}_{3} \mathrm{Cl}$ at polar latitudes resulted to be overestimated, producing unrealistic low values of $\mathrm{CO}$ at low altitudes. Therefore, during the revision process of this paper, we have repeated the analysis substituting the IG2 profile with measurements taken by MLS. In Fig. 15 we present the retrieval obtained for $\mathrm{CO}$ from band D scans. As can be seen in Fig. 15c, the information content of band $\mathrm{D}$ for $\mathrm{CO}$ is not particularly high and low $\mathrm{CO}$ values are found around $13 \mathrm{~km}$ along the whole flight. Low values are also found at lower altitudes for scans from 20 to 29. These values are in reasonably good agreement with the CO data predicted by the CLaMS model, even if the comparison of MARSCHALS CO data with the MLS/Aura ones (an example is reported in Fig. 16a for MARSCHALS scan 11), show that we still find slightly lower values around $150 \mathrm{hPa}$ $(13 \mathrm{~km})$.

In order to further analyse the capability of the MARSCHALS instrument to measure CO profiles, we report here also some results obtained during the mid-latitude test flight performed on 4 November 2009 (and then named TC9 flight). Unfortunately the TC9 flight was performed over an irregular flight path that prevents the optimal use of the measurements acquired in the limb-sounding geometry. In addition the analysis of the spectral response functions of the channeliser measured in the laboratory, performed after the TC9 flight, showed that one of the channels with variable spectral response function was located near the CO line (Spang et al., 2012), and for this reason a channeliser reordering was performed before the PREMIER-Ex Campaign (Sect. 2.2). However in Fig. 16b, we present the results obtained in the analysis of scan 8 . The retrieved $\mathrm{CO}$ profile is in quite good agreement with MLS/Aura version 3.3 coincident data. The results obtained during the TC9 and the PREMIEREx Campaign highlight the MARSCHALS capability of retrieving $\mathrm{CO}$. 

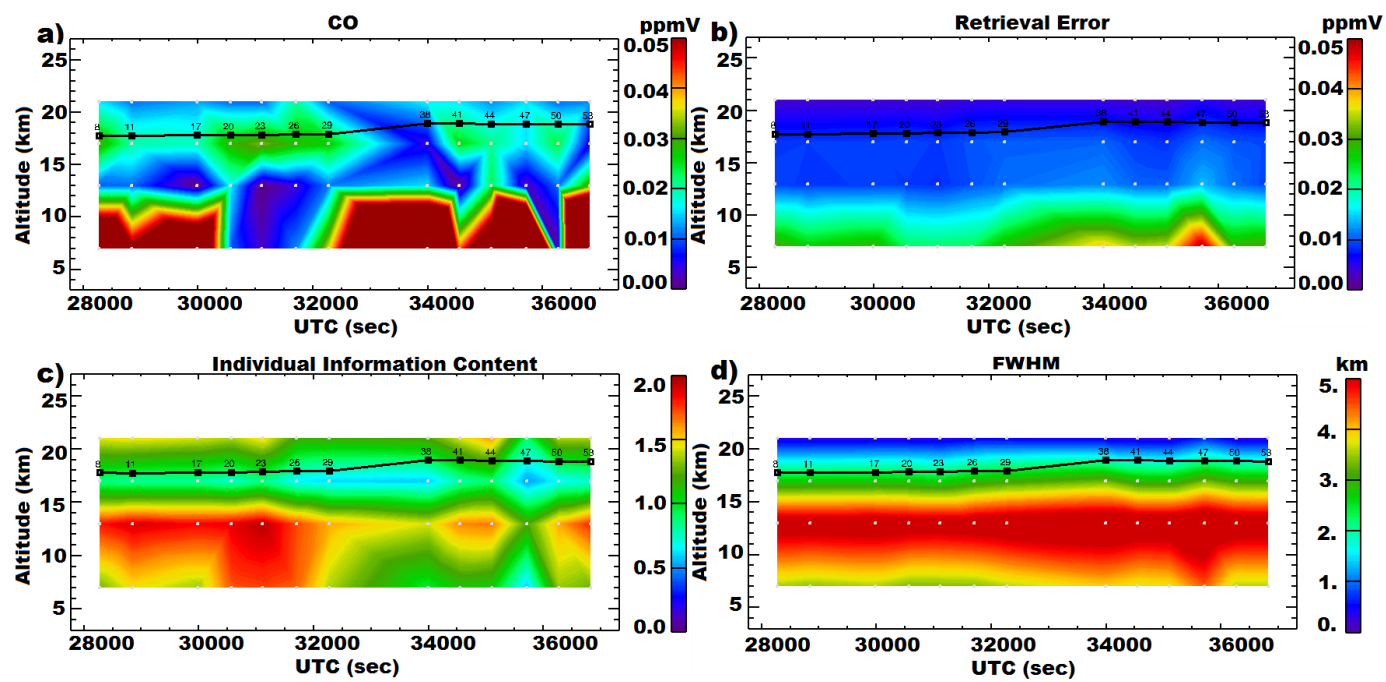

Fig. 15. As in Fig. 5 but for CO retrieved from band D.
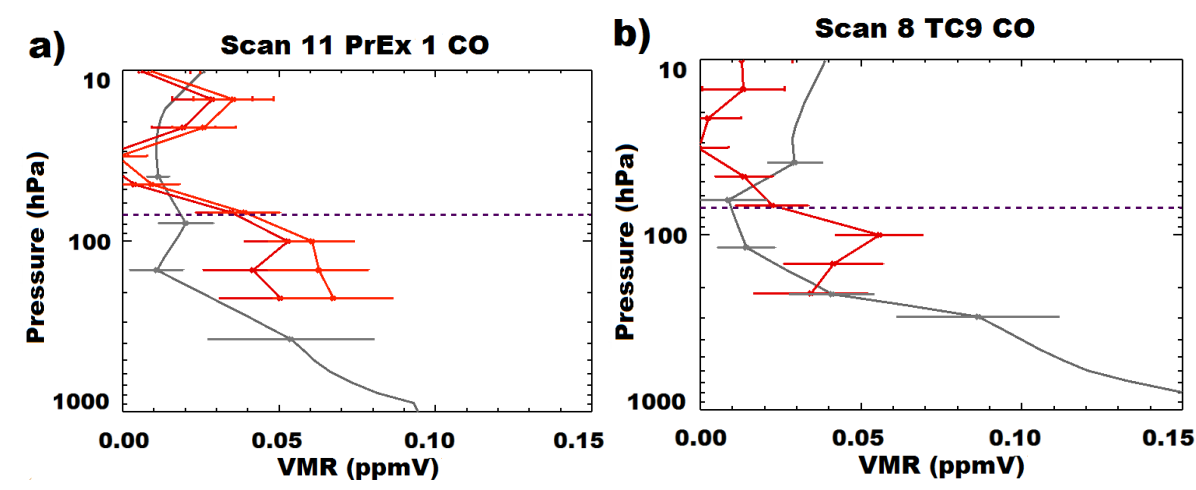

Fig. 16. (a) Comparison of CO profile retrieved from MARSCHALS scan 11 (grey) of PrEx 1 with MLS/Aura (red) CO profiles. (b) Comparison of CO profile retrieved from MARSCHALS scan 8 (grey) of TC9 with MLS/Aura (red) CO profiles. The horizontal dashed line represents the flight altitude level.

\subsection{Clouds}

As already said in Sect. 4.1 to account for possible continuum-like cloud contamination of MARSCHALS spectra we retrieve external continuum profiles (Fig. 17) in the form of a cross section contribution to the spectrum. Since its interpretation is not straightforward, in the following analysis we provide the corresponding extinction coefficient in $\mathrm{km}^{-1}$ obtained from the retrieved external continuum using the ideal gas law.

As can be seen in Fig. 3a, the small values obtained for the reduced $\chi^{2}$ during the flight suggest that the retrieved values of the external continuum were sufficient to model the effects of clouds for almost all the flight. In one case only, for scan number 51, the retrieved extinction coefficient reaches a value $\left(1.4 \times 10^{-3} \mathrm{~km}^{-1}\right.$, slightly higher than the 1. $\times 10^{-3} \mathrm{~km}^{-1}$ threshold, Del Bianco et al., 2007) that suggests the presence of a cloud whose opacity is such to deserve a proper simulation in the retrieval procedure. In this case, the inclusion of the MSSF (Mie Scattering Source Function) module, described in Del Bianco et al. (2007), for the calculation of the scattering contribution given by the cloud should be considered. This module computes the scattering, absorption and extinction coefficients of the cloud and the scattering source function to be included into the radiative transfer equation. This calculation is performed using as input the mean radius $(R)$ and the number density of the cloud particles and some information about the cloud phase (ice/water). Since the retrieved external continuum cannot provide all this information, external information about the cloud properties has to be used. In particular, we used a constant $R$ value and phase taken from the MODIS (MODerate resolution Imaging Spectroradiometer) instrument on-board the Terra satellite, whose measurements are in close temporal coincidence with MARSCHALS measurements $(R=25 \mathrm{mi}-$ cron, ice particles), while the cloud vertical extension (from 

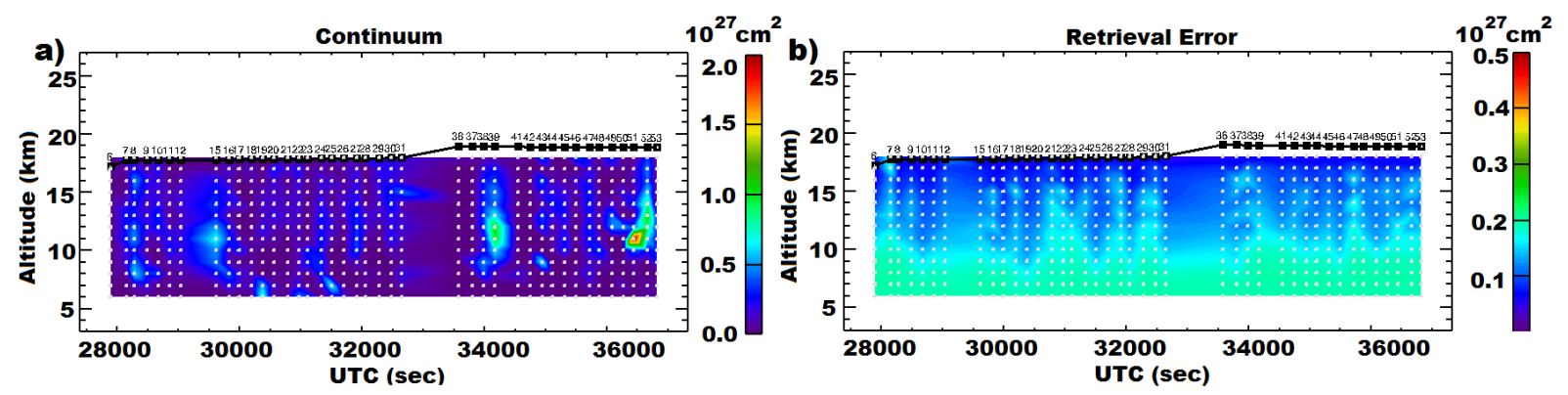

Fig. 17. (a) External continuum retrieved from all bands and (b) its retrieval (biased) error. Units $10^{27} \mathrm{~cm}^{2}$.

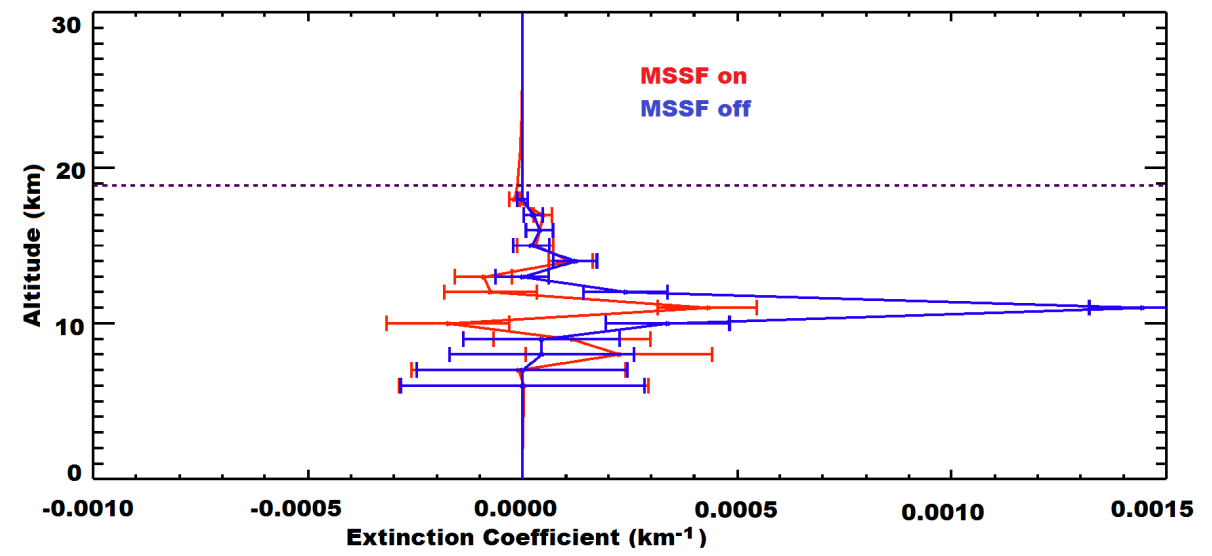

Fig. 18. Extinction coefficient retrieved from MARSCHALS scan 51 with the standard analysis (MSSF module off, in blue) and with MSSF module on (in red). The horizontal dashed line indicates the aircraft altitude during scan acquisition.

12 to $10 \mathrm{~km}$ ) was inferred from the retrieved profiles of the external continuum (see Fig. 18) and the number density $\left(N=0.35 \mathrm{~cm}^{-3}\right)$ was in the range of values observed by insitu instruments on-board the Geophysica during the descent. By repeating the analysis for scan 51 with the MSSF module on and these inputs, we obtain the results displayed in red in Fig. 19.

In Fig. 18, we report the profile of the retrieved extinction coefficient for the standard analysis (blue) and for the analysis performed with the MSSF module on (red). It can be noticed that, when the cloud is simulated into the forward model, the retrieved extinction profile approaches zero, confirming the correct cloud modelling into the MARC FM. The VMR profiles retrieved with the MSSF module on, presented in Fig. 19 in red, show slightly less oscillations compared to the ones retrieved with the MSSF module off (in blue). However, the fact that the external continuum retrieval is sufficient to produce good results is highlighted by the almost identical $\chi^{2}$ values obtained in the case of standard analysis and the analysis with the inclusion of the MSSF module.

The presence of high clouds in the second part of the flight is confirmed by instruments on-board the M-55 Geophysica and on-board the satellites. Information about cloud coverage during the flight is given by the OCM module snapshots, that captured the presence of low clouds during the first leg, no cloud during the second leg and a layer of high altitude clouds on the third leg. This cloud scenario is also confirmed by the MIPAS-STR Cloud Index values (CI, Spang et al., 2004), shown in Fig. 126 of Cortesi et al. (2012). The CI map showed low clouds during the first leg of the flight (Cloud Top Height (CTH) below 9-8 km), no clouds up to $5 \mathrm{~km}$ in the second leg and high altitude clouds (CTH up to $12-11 \mathrm{~km}$ ) in the third leg. Also MODIS and CloudAerosol Lidar and Infrared Pathfinder Satellite Observations (CALIPSO) (Winker et al., 2009) coincident data confirm the presence of high altitude clouds (CTH about $11-12 \mathrm{~km}$ ) in coincidence with MARSCHALS tangent points during the third flight leg. The fact that MIPAS-STR measurements performed in the infrared spectral range were not available below cloud CTH, while the MARSCHALS measurements performed in the millimetre-wave were not affected by cloud presence apart from one scan highlights the importance of the millimetre-wave spectral region to explore the UTLS region. 

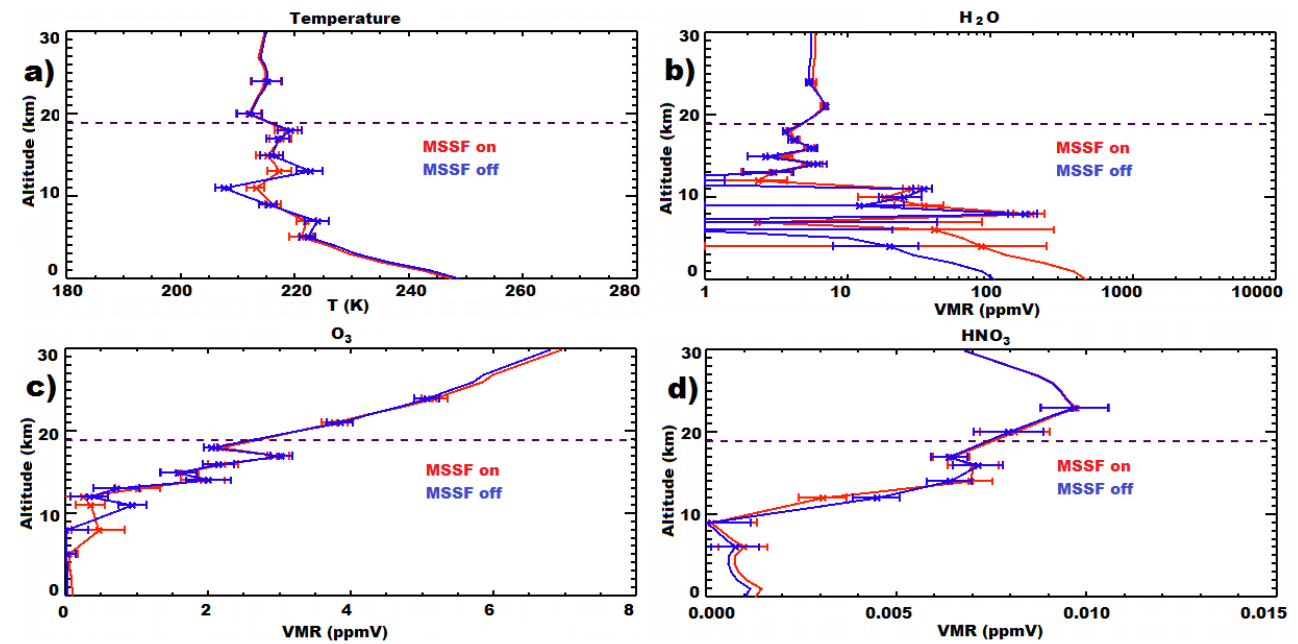

Fig. 19. (a) Temperature, (b) $\mathrm{H}_{2} \mathrm{O}$, (c) $\mathrm{O}_{3}$ and (d) $\mathrm{HNO}_{3}$ retrieved from MARSCHALS scan 51 using (red) or excluding (blue) the MSSF module.

\section{Conclusions}

In this paper, we presented the results from the data analysis of limb sounding observations of the Arctic UTLS, the MARSCHALS data analysis of the PREMIER-Ex flight performed on 10 March from Kiruna, Sweden.

For the first time the instrument was operating in its full configuration with all the three spectral bands working. The availability of all the spectral bands allowed to retrieve the vertical profiles of temperature, water vapour, $\mathrm{O}_{3}, \mathrm{HNO}_{3}, \mathrm{~N}_{2} \mathrm{O}$ and $\mathrm{CO}$, as well as to extract information about the presence of thick clouds. The PREMIER-Ex flight of 10 March 2010 was aimed at studying horizontal mixing processes in the UTLS. The MARSCHALS instrument revealed the presence of a filament of polar air with enhanced $\mathrm{HNO}_{3}$ values, as predicted by the $\mathrm{PV}$ values from ECMWF. The filament from 16 to $14 \mathrm{~km}$ was well traced by the MARSCHALS instrument and the observation backed up by validation with MLS/Aura data. The presence of low and high altitude clouds during the flight did not have a significant impact on MARSCHALS spectra apart from a single scan for which a cloud modelling algorithm was applied for the first time to real data with very good results. The possibility to retrieve CO profile from millimetre-wave spectra was thoroughly explored, since in the PREMIER mission the millimetre-wave sensor STEAM-R was the only instrument able to provide $\mathrm{CO}$ measurements.

As an air-borne demonstrator for the STEAM-R millimetre-wave limb sounder, the MARSCHALS instrument widely demonstrated its capability in this flight. The testing and demonstration of the inverse modelling of MARSCHALS measurements provided crucial information in the perspective of future deployment of millimetre-wave radiometers from space, like the STEAM-R instrument. The quality of the retrieval products obtained from MARSCHALS limb spectra in the Arctic region is, in fact, relevant both for the information content of the individual measurements in the millimetre-wave region, as well as for their potential synergy with the information retrieved from independent and collocated observations in the infrared, like the ones from the InfraRed Limb Sounder (IRLS) of PREMIER.

The potential of combined retrieval of millimetre-wave and middle infrared limb measurements in the UTLS was successfully tested using MARSCHALS measurements reported in this paper and MIPAS-STR data. Full details of this activity will be reported in a paper currently in preparation and are already published in Cortesi et al. (2012).

Acknowledgements. The authors acknowledge ECMWF for access to data. The MLS dataset used in this work were produced with the Giovanni online data system, developed and maintained by the NASA Goddard Earth Sciences (GES) Data and Information Services Center (DISC). The MODIS data used in this study were acquired as part of the NASA's Earth-Sun System Division and archived and distributed by the MODIS Adaptive Processing System (MODAPS). The CALIOP data were obtained from the NASA Langley Research Center Atmospheric Science Data Center. We acknowledge J. Langen for useful comments. This activity was performed as part of the project "PREMIER Analysis of Campaign Data" under ESA contract N. 4000101374//10/NL/CT. The authors gratefully acknowledge all the PIs of the instruments on-board the M-55 Geophysica during the PREMIER-Ex campaign.

Edited by: T. von Clarmann 


\section{References}

Arnone, E., Castelli, E., Papandrea, E., Carlotti, M., and Dinelli, B. M.: Extreme ozone depletion in the 2010-2011 Arctic winter stratosphere as observed by MIPAS/ENVISAT using a 2D tomographic approach, Atmos. Chem. Phys., 12, 9149-9165, doi:10.5194/acp-12-9149-2012, 2012.

Barath, F. T., Chavez, M. C., Cofield, R. E., Flower, D. A., Frerking, M. A., Gram, M. B., Harris, W. M., Holden, J. R., Jarnot, R. F., Kloezeman, W. G., Klose, G. J., Lau, G. K., Loo, M. S., Maddison, B. J., Mattauch, R. J., McKinney, R. P., Peckham, G. E., Pickett, H. M., Siebes, G., Soltis, F. S., Suttie, R. A., Tarsala, J. A., Waters, J. W., and Wilson W. J.: The upper atmosphere research satellite microwave limb sounder instrument, J. Geophys. Res., 98, 10751-10762, doi:10.1029/93JD00798, 1993.

Bianchini, G., Carli, B., Cortesi, U., Del Bianco, S., Gai, M., and Palchetti, L.: Test of far-infrared atmospheric spectroscopy using wide-band balloon-borne measurements of the upwelling radiance, J. Quant. Spectrosc. Ra., 109, 1030-1042, doi:10.1016/j.jqsrt.2007.11.010, 2008.

Carli, B., Bazzini, G., Castelli, E., Cecchi-Pestellini, C., Del Bianco, S., Dinelli, B. M., Gai, M., Magnani, L., Ridolfi, M., and Santurri, L.: MARC: a code for the retrieval of atmospheric parameters from millimetre-wave limb measurements, J. Quant. Spectrosc. Ra., 105, 476-491, doi:10.1016/j.jqsrt.2006.11.011, 2007.

Carlotti, M.: Global-fit approach to the analysis of limb-scanning atmospheric measurements, Appl. Optics, 27, 3250-3254, doi:10.1364/AO.27.003250, 1988.

Christensen, T., Knudsen, B. M., Streibel, M., Andersen, S. B., Benesova, A., Braathen, G., Claude, H., Davies, J., De Backer, H., Dier, H., Dorokhov, V., Gerding, M., Gil, M., Henchoz, B., Kelder, H., Kivi, R., Kyrö, E., Litynska, Z., Moore, D., Peters, G., Skrivankova, P., Stübi, R., Turunen, T., Vaughan, G., Viatte, P., Vik, A. F., von der Gathen, P., and Zaitcev, I.: Vortex-averaged Arctic ozone depletion in the winter 2002/2003, Atmos. Chem. Phys., 5, 131-138, doi:10.5194/acp-5-131-2005, 2005.

Cortesi, U., Del Bianco, S., Gai, M., Dinelli, B. M., Castelli, E., Gerber, D., Oelhaf, H., and Woiwode, W.: PREMIER Analysis of Campaign Data - ESA-ESTEC Contract 4000101374/NL/10/CT, TSRR Vol. 4, edited by: IFAC-CNR, available at: http://www.ifac.cnr.it/ (last access: 23 March 2013), link Editorial Activities - TSRR Vol. 34, 2012.

Del Bianco, S., Carli, B., Cecchi-Pestellini, C., Dinelli, B. M., Gai, M., and Santurri, L.: Retrieval of minor constituents in a cloudy atmosphere with remote-sensing millimetre-wave measurements, Q. J. Roy. Meteorol. Soc., 133, 163-170, 2007.

Dinelli, B. M., Alpaslan, D., Carlotti, M., Magnani, L., and Ridolfi, M.: Multi-target retrieval (MTR): the simultaneous retrieval of pressure, temperature and volume mixing ratio profiles from limb-scanning atmospheric measurements, J. Quant. Spectrosc. Ra., 84, 141-157, 2004.

Dinelli, B. M., Castelli, E., Carli, B., Del Bianco, S., Gai, M., Santurri, L., Moyna, B. P., Oldfield, M., Siddans, R., Gerber, D., Reburn, W. J., Kerridge, B. J., and Keim, C.: Technical Note: Measurement of the tropical UTLS composition in presence of clouds using millimetre-wave heterodyne spectroscopy, Atmos. Chem. Phys., 9, 1191-1207, doi:10.5194/acp-9-1191-2009, 2009.
Dinelli, B. M., Arnone, E., Brizzi, G., Carlotti, M., Castelli, E., Magnani, L., Papandrea, E., Prevedelli, M., and Ridolfi, M.: The MIPAS2D database of MIPAS/ENVISAT measurements retrieved with a multi-target 2-dimensional tomographic approach, Atmos. Meas. Tech., 3, 355-374, doi:10.5194/amt-3-355-2010, 2010.

ESA: Report for Mission Selection: PREMIER, ESA SP-1324/3 (3 volume series), European Space Agency, Noordwijk, the Netherlands, 2012.

Friedl-Vallon, F., Riese, M., Maucher, G., Lengel, A., Hase, F., Preusse, P., and Spang, R.: Instrument concept and preliminary performance analysis of GLORIA, Adv. Space Res., 37, 22872291, doi:10.1016/j.asr.2005.07.075, 2006.

Gettelman, A., Hoor, P., Pan, L. L., Randel, W. J., Hegglin, M. I., and Birner, T.: The extratropical upper troposphere and lower stratosphere, Rev. Geophys., 49, RG3003, doi:10.1029/2011RG000355, 2011.

Hurst, D. F., Oltmans, S. J., Vömel, H., Rosenlof, K. H., Davis, S. M., Ray, E. A., Hall, E. G., and Jordan, A. F.: Stratospheric water vapor trends over Boulder, Colorado: analysis of the 30-yr Boulder records, J. Geophys. Res., 116, D02306, doi:10.1029/2010JD015065, 2011.

Kuhn, T., Tsujimaru, S., Buehler, S., and Verdes, C.: Characterization of millimetre-wave spectroscopic signatures, Technical Report Contract No. 16377/02/NL/FF, European Space Agency (ESA), ESTEC, 2003.

Lacis, A. A., Wuebbles, D. J., and Logan, J. A.: Radiative forcing of climate by changes in the vertical distribution of ozone, J. Geophys. Res., 95, 9971-9981, doi:10.1029/JD095iD07p09971, 1990.

Levenberg, K.: A method for the solution of certain problems in least squares, Q. Appl. Math., 2, 164-168, 1944.

Livesey, N. J., Read, W. G., Froidevaux, L., Waters, J. W., Santee, M. L., Pumphrey, H. C., Wu, D. L., Shippony, Z., and Jarnot, R. F.: The UARS Microwave Limb Sounder version 5 dataset: theory, characterization and validation, J. Geophys. Res., 108, 4378, doi:10.1029/2002JD002273, 2003.

Livesey, N. J., Van Snyder, W., Read, W. G., and Wagner P. A.: Retrieval algorithms for the EOS Microwave Limb Sounder (MLS) instrument, IEEE T. Geosci. Remote, 44, 1144-1155, 2006.

Manney, G. L., Santee, M. L., Rex, M., Livesey, N. J., Pitts, M. C., Veefkind, P., Nash, E. R., Wohltmann, I., Lehmann, R., Froidevaux, L., Poole, L. R., Schoeberl, M. R., Haffner, D. P., Davies, J., Dorokhov, V., Gernandt, H., Johnson, B., Kivi, R., Kyrö, E., Larsen, N., Levelt, P. F., Makshtas, A., McElroy, C. T., Nakajima, H., Parrondo, M. C., Tarasick, D. W., von der Gathen, P., Walker, K. A., and Zinoviev, N. S.: Unprecedented Arctic ozone loss in 2011, Nature, 478, 469-475, doi:10.1038/nature10556, 2011.

Mariotti, A., Moustaoui, M., Legras, B., and Teitelbaum, H.: Comparison between vertical ozone soundings and reconstructed potential vorticity maps by contour advection with surgery, J. Geophys. Res., 102, 6131-6142, doi:10.1029/96JD03509, 1997.

Marquardt, D. W.: An algorithm for the least-squares estimation of nonlinear parameters, SIAM, J. Appl. Math., 11, 431-441, 1963.

Mason, P.: A Review of the 2010 update of the Implementation Plan for the Global Observing System for Climate in Support of the UNFCCC, GCOS SC-XVIII, Doc. 13, 31 July, 2010. 
Moyna, B., Oldfield, M., Gerber, D., Rea, S., Siddans, R., and Kerridge, B.: Technical Report: UAMS Upgrades of MARSCHALS - Technical Data Pack, Final Report of ESA Contract 21990/08/NL/EL "Upgrades to the MARSCHALS Airborne Millimetre/Submillimetre Wave Limb Sounder”, 23 November, 2010.

Murtagh, D., Frisk, U., Merino, F., Ridal, M., Jonsson, A., Stegman, J., Witt, G., Eriksson, P., Jiménez, C., Megie, G., de la Noë, J., Ricaud, P., Baron, P., Pardo, J. R., Hauchcorne, A., Llewellyn, E. J., Degenstein, D. A., Gattinger, R. L., Lloyd, N. D., Evans, W. F. J., McDade, I. C., Haley, C. S., Sioris, C., von Savigny, C., Solheim, B. H., McConnell, J. C., Strong, K., Richardson, E. H., Leppelmeier, G. W., Kyröla, E., Auvinen, H., and Oikarinen, L.: An overview of the Odin atmospheric mission, Can. J. Phys., 80, 309-319, 2002.

Olberg, M., Frisk, U., Lecacheux, A., Olofsson, A. O. H., Baron, P., Bergman, P., Florin, G., Hjalmarson, A., Larsson, B., Murtagh, D. P., Olofsson, G., Pagani, L., Sandqvist, A., Teyssier, D., Torchinsky, S. A., and Volk, K.: The Odin satellite: II. Radiometer data processing and calibration, Astron. Astrophys., 402, 35-38, doi:10.1051/0004-6361:20030336, 2003.

Oldfield, M., Moyna, B., Allouis, E., Brunt, R., Cortesi, U., Ellison, B., Eskell, J., Forward, T., Jones, T., Lamarre, D., Langen, J., de Maagt, P., Matheson, D., Morgan, I., Reburn, J., and Siddans, R.: MARSCHALS: development of an airborne millimetre wave limb sounder, SPIE, 4540, 221-228, 2001.

Piesch, C., Gulde, T., Sartorius, C., Friedl-Vallon, F., Seefeldner, M., Wölfel, M., Blom, C. E., and Fischer, H.: Design of a MIPAS instrument for high-altitude aircraft, Proc. of the 2nd Internat. Airborne Remote Sensing Conference and Exhibition, ERIM, Ann Arbor, MI, Vol. II, 199-208, 1996.

Remedios, J. J., Leigh, R. J., Waterfall, A. M., Moore, D. P., Sembhi, H., Parkes, I., Greenhough, J., Chipperfield, M.P., and Hauglustaine, D.: MIPAS reference atmospheres and comparisons to V4.61/V4.62 MIPAS level 2 geophysical data sets, Atmos. Chem. Phys. Discuss., 7, 9973-10017, doi:10.5194/acpd-79973-2007, 2007.

Ridolfi, M. and Sgheri, L.: A self-adapting and altitude-dependent regularization method for atmospheric profile retrievals, Atmos. Chem. Phys., 9, 1883-1897, doi:10.5194/acp-9-1883-2009, 2009

Rodgers, C. D.: Inverse Methods for Atmospheric Sounding: Theory and Practice, Series on Atmospheric, Oceanic and Planetary Physics, Vol. 2, World Scientific Publishing Co., Singapore, 65$100,2000$.

Sandor, B. J., Read, W. G., Waters, J. W., and Rosenlof, K. H.: Seasonal behavior of tropical to midlatitude upper tropospheric water vapor from UARS MLS, J. Geophys. Res., 103, 2593525947, doi:10.1029/98JD02272, 1998.
Solomon, S., Rosenlof, K. H., Portmann, R. W., Daniel, J. S., Davis, S. M., Sanford, T. J., and Plattner, G.-K.: Contributions of stratospheric water vapor to decadal changes in the rate of global warming, Science, 327, 1219-1223, 2010

Spang, R., Remedios, J. J., and Barkley, M. P.: Colour indices for the detection and differentiation of cloud types in infra-red limb emission spectra, Adv. Space Res., 33, 1041-1047, 2004.

Spang, R., Stroh, F., von Hobe, M., Gerber, D., Moyna, B., Oldfield, M., Rea, S., Reburn, J., Siddans, R., Kerridge, B., Oelhaf, H., and Woiwode, W.: Data Acquisition Report of the PremierEX Scientific Flights, Final Report for ESTEC Contract No. 22670/09/NL/CT “PREMIER Experiment”, 25 May, 2012.

Urban, J., Lautié, N., Le Flochmoën, E., Jiménez, C., Eriksson, P., de La Nöe, J., Dupuy, E., El Amraoui, L., Frisk, U., Jégou, F., Murtagh, D., Olberg, M., Ricaud, P., Camy-Peyret, C., Dufour, G., Payan, S., Huret, N., Pirre, M., Robinson, A. D., Harris, N. R. P., Bremer, H., Kleinböl, A., Küllmann, K., Künzi, K., Kuttippurath, J., Ejiri, M. K., Nakajima, H., Sasano, Y., Sugita, T., Yokota, T., Piccolo, C., Raspollini, P., and Ridolfi, M.: Odin/SMR limb observations of stratospheric trace gases: validation of $\mathrm{N}_{2} \mathrm{O}$, J. Geophys. Res., 110, D09301, doi:10.1029/2004JD005394, 2005a.

Urban, J., Lautié, N., Le Flochemoën, E., Jiménez, C., Eriksson, P., de la Noë, J., Dupuy, E., Ekström, M., El Amraoui, L., Frisk, U., Murtagh, D., Olberg, M., and Ricaud, P.: Odin/SMR limb observations of stratospheric trace gases: level 2 processing of $\mathrm{ClO}, \mathrm{N}_{2} \mathrm{O}, \mathrm{HNO}_{3}$, and $\mathrm{O}_{3}$, J. Geophys. Res., 110, D14307, doi:10.1029/2004JD005741, 2005b.

Waters, J. W., Froidevaux, L., Harwood, R. S., Jarnot, R. F., Pickett, H. M., Read, W. G., Siegel, P. H., Cofield, R. E., Filipiak, M. J., Flower, D. A., Holden, J. R., Lau, G. K., Livesey, N. J., Manney, G. L., Pumphrey, H. C., Santee, M. L., Wu, D. L., Cuddy, D. T., Lay, R. R., Loo, M. S., Perun, V. S., Schwartz, M. J., Stek, P. C., Thurstans, R. P., Boyles, M. A., Chandra, K. M., Chavez, M. C., Chen, G. S., Chudasama, B. V., Dodge, R., Fuller, R. A., Girard, M. A., Jiang, J. H., Jiang, Y., Knosp, B. W., LaBelle, R. C., Lam, J. C., Lee, K. A., Miller, D., Oswald, J. E., Patel, N. C., Pukala, D. M., Quintero, O., Scaff, D. M., Van Snyder, W., Tope, M. C., Wagner, P. A., and Walch, M. J.: The Earth Observing System Microwave Limb Sounder (EOS MLS) on the Aura satellite, IEEE T. Geosci. Remote, 44, 1075-1092, 2006.

Winker, D. M., Vaughan, M. A., Omar, A., Hu, Y., Powell, K. A., Liu, Z., Hunt, W. H., and Young, S. A. Overview of the CALIPSO mission and CALIOP data processing algorithms, J. Atmos. Ocean. Tech., 26, 2310-2323, doi:10.1175/2009JTECHA1281.1, 2009. 